Homology, Homotopy and Applications, vol.11(2), 2009, pp.133-160

\title{
ON THE EMBEDDING DIMENSION OF 2-TORSION LENS SPACES
}

\author{
JESÚS GONZÁLEZ, PETER LANDWEBER AND THOMAS SHIMKUS
}

(communicated by Donald M. Davis)

\begin{abstract}
Using the ku- and BP-theoretic versions of Astey's cobordism obstruction for the existence of smooth Euclidean embeddings of stably almost complex manifolds, we prove that, for $e$ greater than or equal to $\alpha(n)$, the $(2 n+1)$-dimensional $2^{e}$ torsion lens space cannot be embedded in Euclidean space of dimension $4 n-2 \alpha(n)+1$. (Here $\alpha(n)$ denotes the number of ones in the dyadic expansion of a positive integer $n$.) A slightly restricted version of this fact holds for $e<\alpha(n)$. We also give an inductive construction of Euclidean embeddings for $2^{e}$-torsion lens spaces. Some of our best embeddings are within one dimension of being optimal.
\end{abstract}

\section{Main results}

For a positive integer $m$ let $\nu(m)$ and $\alpha(m)$ denote, respectively, the exponent in the highest power of 2 dividing $m$, and the number of ones in the dyadic expansion of $m$. Let $L^{2 m+1}\left(2^{e}\right)$ stand for the $(2 m+1)$-dimensional $2^{e}$-torsion lens space, the quotient of $S^{2 m+1}$ by the standard (diagonal) action of $\mathbb{Z} / 2^{e}$ (viewed as a subgroup of the unit circle $S^{1}$ ).

Unless explicitly noted otherwise, all (non)embedding results are to be understood in the smooth sense. Yet, except for a few low-dimensional cases (carefully pinpointed in the text), our results are within Haefliger's metastable range $2 m \geq 3(n+1)$ where, for a smooth closed manifold $M^{n}$, the existence of a topological embedding $M^{n} \subset \mathbb{R}^{m}$ is equivalent to the existence of a smooth embedding $M^{n} \subset \mathbb{R}^{m}$.

We study the (Euclidean) embedding dimension of $L^{2 m+1}\left(2^{e}\right)$, that is, the dimension of the smallest Euclidean space where this manifold can be embedded. We get no new (non)embeddings for $e=1$ (although we reconstruct some of the known optimal ones), as our methods are generalizations of ideas already used for real projective spaces. Instead, we seek to understand the role the exponent $e$ plays in determining the Euclidean embedding dimension of $L^{2 m+1}\left(2^{e}\right)$.

Our first result is the following analogue of Astey's nonembedding theorem for real projective spaces.

Received June 20, 2009, revised July 16, 2009; published on November 11, 2009.

2000 Mathematics Subject Classification: 57R40, 19L41, 55S45.

Key words and phrases: Euclidean embeddings of lens spaces, connective complex $K$-theory, Brown-

Peterson theory, Euler class, modified Postnikov towers.

This article is available at http://intlpress.com/HHA/v11/n2/a7

Copyright (C) 2009, International Press. Permission to copy for private use granted. 
Theorem 1.1. Let $n$ and $e$ be positive integers with

$$
e \geq \min \left\{\alpha(n)-6, \alpha(n)+1-2^{\nu(n)}\right\},
$$

and set $\delta=\max \{0, \alpha(n)-e\}$. Then the lens space $L^{2(n+\delta)+1}\left(2^{e}\right)$ does not admit an embedding in $\mathbb{R}^{4 n-2 \alpha(n)+1}$.

The more pleasant situation holds for $\delta=0$ (what we call a high-torsion lens space), where (1) holds for free, with Theorem 1.1 affirming the impossibility of embedding $L^{2 n+1}\left(2^{e}\right)$ in $\mathbb{R}^{4 n-2 \alpha(n)+1}$. For $\delta>0$ (a low-torsion lens space), the conclusion is similar, but besides the extra ${ }^{1}$ hypothesis (1), one needs to add the little $(2 \delta)$-correcting term to the dimension of the lens space.

Theorem 1.1 is better (and usually much stronger) than previous nonembedding results with $e \geq 2$. For instance, the best nonembedding that [30] gives for $L^{2 n+1}\left(2^{e}\right)$ (using Atiyah's $\gamma$-operations) is in dimension roughly $3 n+1$. Notable exceptions are the (non)immersion results in $[\mathbf{2 1}, \mathbf{4 7}$ ) (but Theorem 1.1 applies for any $\alpha(n)$ ).

As for positive results, we start by recalling that the Haefliger-Hirsch-MasseyPeterson (HHMP) general embedding result ([38], see also $[\mathbf{1 3}]$ for the case of odd $n$ ) gives an embedding $L^{2 n+1}\left(2^{e}\right) \subset \mathbb{R}^{4 n+1}$ for any pair $(n, e)$. Theorem 1.2 below shows this is, in fact, optimal when $n$ is a power of 2 (improving, in such a case, Theorem 1.1 by one dimension). Theorem 1.3 deals with cases where $n$ is not a power of 2 .

Theorem 1.2. For any e, the HHMP-type embedding $L^{2 n+1}\left(2^{e}\right) \subset \mathbb{R}^{4 n+1}$ is optimal when $\alpha(n)=1$.

Theorem 1.3. There are embeddings of the form $L^{2 n+1}\left(2^{e}\right) \subset \mathbb{R}^{d}$ for the triples $(e, n, d)$ indicated by the columns of Table 1, as well as for the special type of triples $(e, n, d)=(\leq 2,7,26)$. In Table $1, \delta(1)=7, \delta(2)=9$, and $\delta(e)=10$ for $e \geq 3$.

\begin{tabular}{|c|l|l|l|l|}
\hline$e$ & $\geq 1$ & $\geq 1$ & $\geq 1$ & $\geq 1$ \\
\hline$n$ & $2 \ell+1 ; \quad \ell \geq 1$ & $2 \ell+1 ;$ even $\ell, \alpha(\ell) \geq 2$ & $4 \ell+3, \quad \ell \geq 2$ & $4 \ell+3 ; \quad$ even $\ell, \alpha(\ell) \geq 2$ \\
\hline$d$ & $8 \ell+3$ & $8 \ell+2$ & $16 \ell+\delta(e)$ & $16 \ell+\delta(e)-1$ \\
\hline eff & 3 & 4 & 5 & 6 \\
\hline
\end{tabular}

Table 1: $L^{2 n+1}\left(2^{e}\right) \subset \mathbb{R}^{d}$ (last row indicates embedding efficiency, eff, for $e=2$ )

The only potentially non-smooth embeddings (outside Haefliger's range) in this result are those with $\ell=1$ in the second column of Table 1 , that is, the embeddings $L^{7}\left(2^{e}\right) \subset \mathbb{R}^{11}$. On the other hand, the case $\ell=0$ is indeed exceptional; as indicated in Remark 2.4, all 3-dimensional lens spaces (smoothly) embed in $\mathbb{R}^{5}$, but no 3 -dimensional lens space embeds in $\mathbb{R}^{4}$.

The strength of Theorem 1.3 is better appreciated by contrasting it with Theorem 1.1. For instance, in case of high-torsion lens spaces, the embedding given in

\footnotetext{
${ }^{1}$ Motivated by the situation for $e=1$ in $[\mathbf{2}]$, it was conjectured in [19] that the hypothesis (1) can
} be removed in the low-torsion case. 
column $c$ of Table 1 is within $d(c)$ dimensions of being optimal, where

$$
d(c)=(2 \alpha(\ell)-1,2 \alpha(\ell)-2,2 \alpha(\ell), 2 \alpha(\ell)-1)
$$

for $c=(2,3,4,5)$. These figures lead to a couple of interesting comments about the potential optimality of our results. Firstly, in terms of the efficiency eff of an embed$\operatorname{ding} M^{m} \subset \mathbb{R}^{2 m-\text { eff }}$, we observe that, although the embeddings in columns $c=4,5$ have better efficiency than those for $c=2,3,(2)$ indicates that the latter ones are, in general, closer to being optimal. This is of course interpreted as saying that the difficulty in computing the embedding dimension of $L^{2 n+1}\left(2^{e}\right)$ increases with $\alpha(n)$, a standard empirical fact for real projective spaces. Secondly, note that the only situation where $d(c)$ could be zero - thus indicating an optimal embedding - is for $c=3$ and $\alpha(\ell)=1$; unfortunately the last equality is ruled out for $c=3$. In Remark 6.4 we discuss the expectations for what the optimal embedding could turn out to be for this situation (see also Remark 6.6 and Example 6.7, the difficulty of the former being the first major obstacle in the field, and the main motivation for this paper). Now, at the opposite extreme of 2-torsion (i.e., for real projective spaces), we point out (Remark 4.11) that the cases with the lowest allowed value of $\alpha(\ell)$ in the fourth column of Table 1 are known to give optimal embeddings for $e=1$. Likewise, the cases with the lowest allowed value of $\alpha(\ell)$ in the fifth column of Table 1 give currently best known embeddings for $e=1$.

We now comment on the methods (their origins and expectations) used in proving the three theorems above.

Following Astey's work [2] for real projective spaces, the proof of Theorem 1.1 extends, to the embedding realm, the nonimmersion results for lens spaces in $[\mathbf{1 8}$, 19]. In turn, these arose from Davis' strong nonimmersion result for real projective spaces [5] (see also [1]). The form of all these results combines Euclidean dimension, manifold dimension, and (for lens spaces) torsion in the fundamental group, in order to better reflect irregularities in the immersion and (now) embedding dimensions. For real projective spaces, ${ }^{2}$ the strength of nonimmersion and nonembedding results of this type is due to the fact that, while involving a simple but general statement, they are often either the currently best known, or within a short distance of the best known. For instance, as originally explained in [5] and updated in [4], whenever it is currently known that $\mathrm{P}^{n}$ does not immerse in $\mathbb{R}^{m}$, then [5] affirms that $\mathrm{P}^{n+i}$ does not immerse in $\mathbb{R}^{m-j}$ for some nonnegative integers $i$ and $j$ with $i+j \leq 3$ (the last inequality can be improved to $i+j \leq 2$, if one excludes the nonimmersion results in $[\mathbf{1 2}])$. Such a comparison takes into account the recent works $[\mathbf{4}, \mathbf{1 1}, \mathbf{2 8}, \mathbf{2 9}]$ obtained with some of the most sophisticated homotopy technology currently available. One should keep in mind, though, that for a fixed projective space, current nonimmersion results can improve the original [5] by arbitrarily large Euclidean dimensions - but this is not new: the much older [27] has the same effect over [5]. At any rate, it is interesting to keep in mind the possibility that the recent advances in homotopy methods could turn out to be particularly helpful in settling some of the small gaps pinpointed in (2).

Theorem 1.2 is not really new; as we will see in Section 3, it follows from the exact same argument used in $[\mathbf{3 3}]$ for the case $e=1$ (see also [31]).

\footnotetext{
${ }^{2}$ Technical problems prevented the first author from obtaining in $[\mathbf{1 9}]$ the required general result that
} would have made these considerations work for lens spaces. 
The proof of Theorem 1.3 adapts, to lens spaces, the inductive Euclidean embedding constructions done in $[\mathbf{3 6}]$ for real projective spaces $\mathrm{P}^{n}$. We need to proceed with care though, so as to avoid the flaw in [36] coming from using the (not properly argued) immersions in $[\mathbf{1 5}, \mathbf{1 6}]$ (see Remarks 4.7 and 4.8). One of the main ingredients in [36] comes from Milgram's linear algebra techniques in [39] for constructing, over a given space $\mathrm{P}^{n+k}$, spanning fields with the property that enough of them are trivial over a given smaller $\mathrm{P}^{n}$. But for lens spaces we need to replace such a linear algebra component by a careful obstruction theory analysis in the form of modified Postnikov towers. As a side consequence we get the fact that Milgram's linear algebra input in the (start of the) inductive method in [36] not only has the power to produce, when combined with the embeddings for $\mathrm{P}^{7}$ and $\mathrm{P}^{15}$ in [44], many optimal embeddings of real projective spaces (see Remark 4.11), but can actually be extended, via obstruction theory, to the fatter ${ }^{3}$ lens spaces in order to trigger a reasonably strong (and in a sense optimal - see the final remarks in Section 5) inductive construction of their embeddings. In this respect, it is to be noted that not all known methods for constructing embeddings of real projective spaces have a chance to work for higher 2 -torsion lens spaces. Indeed, some of the projective space arguments known to date use, in an essential way, phenomena inherent to real projective spaces, thus constructing low-dimensional embeddings that, in general, will not have to be true for hightorsion lens spaces. So, part of the problem consists of identifying methods that do construct (reasonably strong) embeddings for general $2^{e}$-torsion lens spaces. This is indeed one of the goals set for this paper, with Proposition 5.5 its most notable accomplishment.

Observe that having concentrated in this paper on lens spaces of torsion a power of 2 is not a real restriction. For one, as we already mentioned, except for a few lowdimensional cases, all of our results are within Haefliger's metastable range. Therefore, using the canonical projection $L^{2 m+1}\left(2^{e}\right) \rightarrow L^{2 m+1}\left(2^{e} k\right)$ in [45, Theorem B], we see that, for odd $k$, our embedding results for $L^{2 m+1}\left(2^{e}\right)$ automatically apply to $L^{2 m+1}\left(2^{e} k\right)$.

The paper is organized as follows. Theorem 1.1 is proved in Section 2 by a straightforward application of Astey's general MU-obstruction result [2] for Euclidean embeddings of stably almost complex manifolds, together with the ku-calculations in [18] and the BP-calculations in [19]. Section 3 is devoted to the proof of Theorem 1.2, and to the description of some easy embedding results, mainly setting grounds for comparing with the embeddings in Section 4. The ideas in [36] are worked out in Section 4 from the point of view of lens spaces in order to prove Theorem 1.3. Section 5 gives the obstruction theory details that replace Milgram's linear algebra input. The goal of the final Section 6 is to pinpoint key subtleties arising when we compare the behavior of the (immersion and) embedding dimensions of $2^{e}$-torsion lens spaces, as $e$ varies.

\section{Acknowledgements}

This research was completed while the first author was on a sabbatical break visiting CIMAT, in Guanajuato, México. The first author thanks faculty and staff at CIMAT

\footnotetext{
${ }^{3}$ Section 6 discusses the sense in which $2^{e}$-torsion lens spaces get fatter as $e$ increases.
} 
for creating the conditions that led to an enjoyable and productive visit. A CONACyT sabbatical scholarship (290537 CIMAT) provided the required financial support for the visit, while CONACyT Grant 106923 provided partial funds for attending international conferences on the subject.

\section{Proof of Theorem 1.1}

Let $M$ be a smooth compact stably almost complex manifold, with stable normal bundle $\nu$ of complex dimension $d$. Let $h^{*}$ denote a multiplicative complex-oriented cohomology theory whose ring of coefficients is concentrated in even dimensions, and where 2 is not a zero divisor. Let $\xi_{k}$ stand for the canonical real line bundle over the $k$-dimensional real projective space $\mathrm{P}^{k}$. Then, as proved in [2], $M$ does not admit a Euclidean embedding with codimension $2 \ell$ provided the following two conditions hold with $k=d-\ell$ :

(a) The $h^{*}$-Euler class of $\nu \otimes \xi_{2 k}$ is nontrivial.

(b) The external product

$$
h^{\text {even }}(M) \otimes_{h_{*}} h^{*}\left(\mathrm{P}^{2 k}\right) \rightarrow h^{\text {even }}\left(M \times \mathrm{P}^{2 k}\right)
$$

is an isomorphism.

In the case $M=L^{2 m+1}\left(2^{e}\right)$ we take $\nu=\left(2^{N}-m-1\right) \eta$, where $N$ is any large positive integer, and where $\eta$ is obtained as the pull-back, under the canonical projection $L^{2 m+1}\left(2^{e}\right) \rightarrow \mathbb{C P}^{m}$, of the complex Hopf line bundle over the $m$-dimensional complex projective space.

Proof of Theorem 1.1. Under the above conditions, and in order to rule out a possible $(4 n-2 \alpha(n)+1)$-dimensional Euclidean embedding of $L^{2 m+1}\left(2^{e}\right)$, for $m=n+\delta$, the bundle to consider in (a) above is

$$
\left(2^{N}-m-1\right) \eta \otimes \xi_{2 k}
$$

where $k=2^{N}-2 n+\alpha(n)-1$. If we let $h^{*}=\mathrm{ku}^{*}$, connective complex $K$-theory, for $\delta=0$, and $h^{*}=\mathrm{BP}^{*}$, Brown-Peterson theory at the prime 2 , for $\delta>0$, then the main results in [18] (for $\delta=0$ ) and in [19] (for $\delta>0$ ) assert that the $h^{*}$-Euler class of (3) is nontrivial. The result then follows since, in our case $\left(M=L^{2 m+1}\left(2^{e}\right)\right)$, condition (b) above is a restatement of Proposition 3.1 in [18].

Remark 2.1. In [19, Proposition 2.1] it is proved that if $L^{2 m+1}\left(2^{e}\right)$ were to immerse in $\mathbb{R}^{4 n-2 \alpha(n)}$, then the bundle (3) would have a nowhere zero section. Thus, in retrospect, the $h^{*}$-calculations in [18] and [19] simultaneously yield the non-immersibility and non-embeddability of $L^{2 m+1}\left(2^{e}\right)(m=n+\delta$, as in the proof of Theorem 1.1) in Euclidean dimensions $4 n-2 \alpha(n)$ and $4 n-2 \alpha(n)+1$, respectively. (This is one of the reasons why the remarks about Theorem 1.1 in Section 1 mainly concern immersions rather than embeddings.)

Remark 2.2. Astey's nonembedding result is indirectly based on the triviality of the (generalized) Euler class of the normal bundle for an embedding in a Euclidean space (see [40, Corollary 11.4]). Here we point out firstly that, when the above observation is used for singular cohomology with $\mathbb{Z}$ coefficients (rather than ku or BP), we obtain 
the well-known Fact 2.3 below, and secondly that, together with Fact 6.2 in Section 6 , this yields the impossibility of finding a Euclidean embedding with codimension 2 for any $2^{e}$-torsion lens space of dimension $\geq 5$. The only notable possible exception is the parallelizable 7 -dimensional real projective space $\mathrm{P}^{7}$ (see Remark 6.6 at the end of the paper). In particular, we recover cases $n=2,3$ of Theorem 1.1 (for high-torsion lens spaces in the case of $n=3$ ).

Fact 2.3. The only closed smooth orientable manifolds $M$ that can be embedded in Euclidean space with codimension 2 are necessarily stably parallelizable. (As observed in [26], the converse is in general false). Indeed, the normal 2-plane bundle $\nu_{M}$ of such an embedding would be orientable with trivial Euler class. But $\mathrm{SO}(2)=S^{1}$, so that being the realification of a complex line bundle with vanishing first Chern class, $\nu_{M}$ is in fact trivial, and consequently $M$ is stably parallelizable.

Remark 2.4. As for low (i.e., 1 or 2) codimension Euclidean embeddings of lens spaces, Remark 2.2 does not consider the case of lens spaces of dimension 1 or 3 . Luckily, these are well understood. Of course $S^{1}$ embeds optimally in $\mathbb{R}^{2}$. On the other hand, according to $[\mathbf{2 3}]$ no 3 -dimensional lens space embeds into $\mathbb{R}^{4}$; however they all (smoothly) embed in $\mathbb{R}^{5}$, as follows from $[\mathbf{2 5}]$.

\section{Proof of Theorem 1.2 (early methods)}

We have just indicated in Remark 2.4 that the case $n=1$ in Theorem 1.2 is well known.

Proof of Theorem 1.2 for $n>1$. We derive a contradiction from assuming an embed$\operatorname{ding} L^{2 n+1}\left(2^{e}\right) \subset \mathbb{R}^{4 n}$. The argument uses singular cohomology groups with mod 2 coefficients (which will be suppressed from the notation). The Gysin sequence of the normal sphere bundle $E$ (with projection $\pi$ ) for the hypothesized embedding reduces to split short exact sequences

$$
0 \rightarrow H^{q}\left(L^{2 n+1}\left(2^{e}\right)\right) \stackrel{\pi^{*}}{\longrightarrow} H^{q}(E) \stackrel{\psi}{\longrightarrow} H^{q-2 n+2}\left(L^{2 n+1}\left(2^{e}\right)\right) \rightarrow 0
$$

(the homomorphism after $\psi$ is multiplication by the Euler class, which is trivial as mentioned in Remark 2.2). For dimensions $q<4 n-1$, the splitting is made geometrically explicit in $[\mathbf{3 7}$, Section 3$]$ by exhibiting a subalgebra $A^{*}$ such that

(a) $H^{q}(E)=\pi^{*}\left(H^{q}\left(L^{2 n+1}\left(2^{e}\right)\right)\right) \oplus A^{q}$, for $0<q<4 n-1$,

(b) $A^{4 n-1}=0$, and

(c) $A$ is stable under cohomology operations.

Consequently, in the dimensions of $(\mathrm{a}), \psi$ restricts to an isomorphism from $A^{q}$ onto $H^{q-2 n+2}\left(L^{2 n+1}\left(2^{e}\right)\right)$. In particular, there is a well-defined element $a \in A^{2 n-2}$ with $\psi(a)=1$. Furthermore, as observed in [37, page 784], each element $\omega \in H^{*}(E)$ can be expressed uniquely in the form

$$
\omega=\pi^{*}(u)+a \cdot \pi^{*}(v)
$$

where $u, v \in H^{*}(E)$. As a last piece of notation, let $x, y \in H^{*}\left(L^{2 n+1}\left(2^{e}\right)\right)$ stand for the nontrivial classes in dimensions 1 and 2 , respectively. They are connected by the 
relation $\beta_{e}(x)=y$, where $\beta_{e}$ is the Bockstein associated to the extension

$$
0 \rightarrow \mathbb{Z} / 2^{e} \rightarrow \mathbb{Z} / 2^{2 e} \rightarrow \mathbb{Z} / 2^{e} \rightarrow 0
$$

We recall that $H^{*}\left(L^{2 n+1}\left(2^{e}\right)\right)$ is generated by $x$ and $y$ subject to the relations

$$
y^{n+1}=0 \text { and } x^{2}=\varepsilon y
$$

where $\varepsilon=1$ for $e=1$, and $\varepsilon=0$ for $e>1$.

Start with the class $\omega \in A^{2 n-1}$ corresponding to $x$ under $\psi$, which must clearly have the form $\omega=\delta \pi^{*}\left(x y^{n-1}\right)+a \cdot \pi^{*}(x)$, for $\delta \in \mathbb{Z} / 2$. Property (c) above implies that

$$
\delta \pi^{*}\left(y^{n}\right)+a \cdot \pi^{*}(y)=\beta_{e}\left(\delta \pi^{*}\left(x y^{n-1}\right)+a \cdot \pi^{*}(x)\right)
$$

(as in [33], one uses here the fact that $a$ is the mod 2 reduction of a similar integral class) lies in $A$, whereas property (b) above yields

$$
a^{2} \cdot \pi^{*}(x y)=\left(\delta \pi^{*}\left(y^{n}\right)+a \cdot \pi^{*}(y)\right)\left(\delta \pi^{*}\left(x y^{n-1}\right)+a \cdot \pi^{*}(x)\right)=0
$$

(the first equality uses the relation $y^{n+1}=0$, recalling $n>1$ ). On the other hand, $[\mathbf{3 7}$, lemma on page 785] claims that the expression (4) for $a^{2}=\operatorname{Sq}^{2 n-2}(a)$ has the form

$$
a^{2}=\pi^{*}\left(a^{\prime}\right)+a \cdot \pi^{*}\left(\overline{\mathrm{W}}_{2 n-2}\right),
$$

where $\overline{\mathrm{W}}_{2 n-2}$ is the normal Stiefel-Whitney class of $L^{2 n+1}\left(2^{e}\right)$. But a straightforward computation using the hypothesis $\alpha(n)=1$ gives $\overline{\mathrm{W}}_{2 n-2}=y^{n-1}$, so that (6) reduces, by dimensional reasons (once again recall $n>1$ ), to $a \cdot \pi^{*}\left(x y^{n}\right)=0$ or, by the uniqueness of (4), $x y^{n}=0$, in contradiction to (5).

We close this section with a rather geometric argument leading to an easy embedding result for lens spaces, which partially generalizes Mahowald's embedding $\mathrm{P}^{k} \subset$ $\mathbb{R}^{2 k-2}$ (valid for $k>3$, with $k \equiv 3 \bmod 4$; see [34, Theorem 7.2.2]). The (improved) full generalization is given by Theorem 1.3 .

Proposition 3.1. $L^{2 n+1}\left(2^{e}\right)$ embeds in $\mathbb{R}^{4 n}$ provided $n \equiv 3 \bmod 4$.

Proof. This is a consequence of Lemma 3.2 below and the fact proved in [50] that, for $m \equiv 5,6,7 \bmod 8$ and $m \geq 7$, every spin manifold $M^{m}$ embeds in $\mathbb{R}^{2 m-2}$.

Lemma 3.2. $L^{2 n+1}\left(2^{e}\right)$ has a spin structure precisely for $n=0$, and for odd $n \geq 1$.

Proof. Since $L^{2 n+1}\left(2^{e}\right)$ is orientable, everything reduces to a straightforward calculation of the second Stiefel-Whitney class $w_{2}=w_{2}\left(L^{2 n+1}\left(2^{e}\right)\right)$. We present this using Wu's formula $w=\operatorname{Sq}(v)$ ([40, Theorem 11.14]), thus avoiding the need for explicit descriptions of tangent bundles. Wu's first relation gives $v_{1}=w_{1}$, which we already observed to be trivial. Then Wu's second relation reduces to $v_{2}=w_{2}$. Thus, it suffices to observe that $\mathrm{Sq}^{2}$ acts trivially on $H^{2 n-1}\left(L^{2 n+1}\left(2^{e}\right) ; \mathbb{Z} / 2\right)$ precisely when $n$ is odd. (The mod 2 cohomology ring for $L^{2 n+1}\left(2^{e}\right)$ can easily be obtained from [24, Example 3E2], whereas the action of the Steenrod algebra follows from the well-known situation for $\mathbb{C P}^{n}$.) 


\section{Inductive construction of embeddings}

Following the inductive methods in [36], we now produce explicit Euclidean embeddings for $2^{e}$-torsion lens spaces. In this section we use the shorter notation $L_{n, e}$ for $L^{2 n+1}\left(2^{e}\right)$.

For $e \geq 1$, consider $\mathbb{Z} / 2^{e}$ as the subgroup of $S^{1}$ of $2^{e}$ th roots of unity. For $k, j \geq 0$, think of $S^{2(k+j+1)+1}$ as the join of $S^{2 k+1}$ and $S^{2 j+1}$ via the explicit homeomorphism $\phi: S^{2 k+1} \star S^{2 j+1} \rightarrow S^{2(k+j+1)+1}$ sending $(x, y, t)$ into $(\sqrt{1-t} x, \sqrt{t} y)$. Under this identification, the standard $\mathbb{Z} / 2^{e}$-action on $S^{2(k+j+1)+1}$ takes the form

$$
\omega(x, y, t)=(\omega x, \omega y, t) .
$$

By restriction, this yields actions on the subsets $L^{\prime}, R^{\prime} \subset S^{2 k+1} \star S^{2 j+1}$ determined by the conditions $t \leq 1 / 2$ and $t \geq 1 / 2$, respectively. At the orbit space level we get a decomposition

$$
L_{k+j+1, e}=L \cup R
$$

where $L$ (resp., $R$ ) is the normal real disc bundle for the embedding of $L_{k, e}$ (resp., $\left.L_{j, e}\right)$ in $L_{k+j+1, e}$ coming from the first (resp., last) coordinates. Explicit models for $L$ and $R$ are then given by

$$
L=\left(S^{2 k+1} \times D^{2 j+2}\right) / \mathbb{Z} / 2^{e} \text { and } R=\left(D^{2 k+2} \times S^{2 j+1}\right) / \mathbb{Z} / 2^{e},
$$

where, as indicated by (7), the $\mathbb{Z} / 2^{e}$ action is diagonal in both cases. More familiar descriptions are obtained from the following considerations.

Let $H_{m}$ stand for the canonical complex line bundle over $\mathbb{C P}^{m}$, and let $\eta_{m}$ denote the complex conjugate bundle $H_{m}^{*}$. The map $\lambda: S^{2 m+1} \times \mathbb{C} \rightarrow \mathbb{C P}^{m} \times \mathbb{C}^{m+1}$ sending $(x, z)$ into $([x], z x)$, where $[x]$ stands for the complex line determined by $x$, satisfies $\lambda(x, \omega z)=$ $\lambda(\omega x, z)$ for $\omega \in S^{1}$, and produces the standard model for $H_{m}$ as $S^{2 m+1} \times{ }_{S^{1}} \mathbb{C}$ - the orbit space of the $S^{1}$ action on $S^{2 m+1} \times \mathbb{C}$ given by $\omega(x, z)=\left(\omega^{-1} x, \omega z\right)$. In particular, a model for $\eta_{m}$ is given by $\left(S^{2 m+1} \times \mathbb{C}\right) / S^{1}$ (diagonal action now). As a result, letting $\eta_{m, e}$ denote the pull-back of $\eta_{m}$ under the canonical map $L_{m, e} \rightarrow \mathbb{C P}^{m}$, we get that a model for the Whitney multiple $n \eta_{m, e}$ is given by $\left(S^{2 m+1} \times \mathbb{C}^{n}\right) / \mathbb{Z} / 2^{e}$ (diagonal action, of course). Consequently, we have proved:

Lemma 4.1. $L=D\left((j+1) \eta_{k, e}\right)$ and $R=D\left((k+1) \eta_{j, e}\right)$, the total spaces of the disc bundles for (the realifications of) $(j+1) \eta_{k, e}$ and $(k+1) \eta_{j, e}$.

The easy geometric argument in the proof of [49, Lemma 3.1] can now be adapted to the current situation in order to identify

$$
L \cap R=\left(S^{2 k+1} \times S^{2 j+1}\right) /\left(\mathbb{Z} / 2^{e}\right)
$$

(corresponding to $t=1 / 2$ under $\phi$ ) with the sphere bundle of the realification of the (exterior) tensor product $\eta_{k, e}^{*} \otimes \eta_{j, e}$. But in order to directly apply the results in [36], we switch to their sphere bundle and mapping cylinder notation: $L \cap R$ is the total space of both sphere bundles $S\left((j+1) \eta_{k, e}\right)$ and $S\left((k+1) \eta_{j, e}\right)$. Moreover, letting $\pi_{k}: S\left((j+1) \eta_{k, e}\right) \rightarrow L_{k, e}$ and $\pi_{j}: S\left((k+1) \eta_{j, e}\right) \rightarrow L_{j, e}$ stand for the bundle projections, the considerations in (8) and (9) yield the following reinterpretation of Lemma 4.1. 
Lemma 4.2. $L$ and $R$ are, respectively, the mapping cylinders of $\pi_{k}$ and $\pi_{j}$. Likewise, $L_{k+j+1, e}$ is the double mapping cylinder $M\left(\pi_{k}, \pi_{j}\right)$.

Here the notation is as in [36, Section 5]. In particular, Theorems 2.2 and 5.2 in [36] yield an inductive method for constructing embeddings of lens spaces (compare to $[\mathbf{4 3}$, Proposition 1]).

Proposition 4.3. Let $L_{k, e} \subset \mathbb{R}^{\alpha}, L_{j, e} \subset \mathbb{R}^{\beta}$, and $(k+1) \eta_{j, e} \subset \mathbb{R}^{\sigma+\beta}$ be embeddings ${ }^{4}$, and assume that the first embedding admits $\sigma$ everywhere linearly independent normal sections. Then there is a (topological) embedding $L_{k+j+1, e} \subset \mathbb{R}^{\alpha+\beta+1}$, provided either one of the following two numerical conditions holds:

(i) $\sigma+\beta>4 j+2$.

(ii) $\sigma+\beta=4 j+2$ and $2 k+3 \leq 8 a+2^{b}$, where $\nu(2 j+2)=4 a+b$ and $0 \leq b \leq 3$.

Remark 4.4. From (8) and (9) we also get the explicit identifications $(j+1) \eta_{k, e}=$ $L_{k+j+1, e}-L_{j, e}$ and $(k+1) \eta_{j, e}=L_{k+j+1, e}-L_{k, e}$. These are analogous to the models used in [43], a paper exploiting the inductive method coming from Proposition 4.3 to construct Euclidean embeddings for odd-torsion lens spaces. In that case, the Postnikov analysis (corresponding to our rather intricate Section 5) relies on [48] where the obstructions are directly handled by showing the triviality mod 2 of the relevant Pontryagin classes. The reader may want to compare this to our final argument in the proof of Proposition 5.8.

For a fixed $k$, Proposition 4.3 allows us to start an inductive process (which we call a round) for constructing embeddings of lens spaces. Namely, having constructed an embedding $L_{j, e} \subset \mathbb{R}^{\beta}$, this result allows us to deduce the "next" embedding $L_{j+(k+1), e} \subset \mathbb{R}^{\alpha+\beta+1}$ provided $L_{k, e} \subset \mathbb{R}^{\alpha}$ (what we call the triggering ingredient, as it is independent of $j$ ) and $(k+1) \eta_{j, e} \subset \mathbb{R}^{\sigma+\beta}$ (what we call the feeding ingredient, as it has to be verified for each $j$ in the inductive process). The best results are obtained for rounds with $k$ one less than a power of 2 (when $\sigma_{k, e}$ in the next lemma is largest). The following result, a consequence of [36, Theorem 2.2] and the summary of immersions for lens spaces in [21, Section 1], provides us with the triggering ingredient.

Lemma 4.5. There are embeddings $L_{k, e} \subset \mathbb{R}^{4 k+2}$ with $\sigma_{k, e}$ everywhere linearly independent normal sections, for the triples $\left(k, e, \sigma_{k, e}\right)$ indicated by the columns of Table 2.

\begin{tabular}{|c|c|c|c|c|}
\hline$k$ & 3 & 3 & 3 & 1 \\
\hline$e$ & 1 & 2 & $\geq 3$ & $\geq 1$ \\
\hline$\sigma_{k, e}$ & 7 & 5 & 4 & 3 \\
\hline
\end{tabular}

Table 2: Values of $\sigma_{k, e}$

\footnotetext{
${ }^{4}$ Only the first of the three hypothetical embeddings in Proposition 4.3 needs to be smooth.
} 
Remark 4.6. In getting the value $\sigma_{3,1}=7$, one needs to observe that, having a trivial first Stiefel-Whitney class, the normal bundle to an immersion $P^{7} \rightarrow \mathbb{R}^{8}$ is trivial. Similarly, the value $\sigma_{1, e}=3$ can be deduced (for instance) from the fact that the normal bundle $\nu$ to an embedding $L_{1, e} \subset \mathbb{R}^{5}$ (Remark 2.4) is trivial. Indeed, since lens spaces are orientable, one has $w_{1}(\nu)=0$, so that $\nu$ is in fact a (1-dimensional) complex vector bundle. Its triviality then follows since $c_{1}(\nu)=\chi(\nu)$ is also trivial, as noted in the first statement of Remark 2.2.

Remark 4.7. The flaw in [36] - our guiding reference in this section - arises from an improperly supported use of Lemma 4.5. In our notation, it is affirmed in [36] that Lemma 4.5 is true for $k=2^{\mu}-1$ and $\sigma_{k, 1}=2 \mu+\delta$, where

$$
\delta= \begin{cases}1, & \mu \equiv 0,1 \bmod 4 \\ 3, & \mu \equiv 2 \bmod 4 \\ 2, & \mu \equiv 3 \bmod 4\end{cases}
$$

(cases with $\mu=1,2$ are contained in Table 2). For $\mu \geq 3$, such an assertion is deduced in [36] from an immersion $L_{k, 1} \rightarrow \mathbb{R}^{4 k+2-\sigma_{k, 1}}$ — which would be optimal in view of [27]. But such an immersion has been correctly argued only for $3 \leq \mu \leq 5$ (see [6] and [8]; the former has a historical recollection of the problem). The outcome is that only the first five inductive rounds in [36] are properly supported. As a consequence, all the results in Section 1 after Theorem 1.6 in [36] are invalidated, and this goes also for the main theorem appearing at the very start of that paper. Furthermore, the careful reader will realize that Theorem 1.6 - the only "non-approximative" result in [36] - is correctly argued only after fixing a typo: in part (d) one must add the restriction $\alpha(k) \geq 2$ (at any rate, this restricted case was settled much later in [7]). On the other hand, the point in the main result of [36], namely, the fact that the efficiency in the embedding dimension of $\mathrm{P}^{n}$ is an unbounded function of $n$, was settled in a stronger way in $[49]$.

Remark 4.8. The thorough reader will note that the information recollected in $[\mathbf{2 1}]$ would allow us to go for one further round (for $k=7$, where $\sigma_{7,1}=8$ and $\sigma_{7, e}=7$ for $e \geq 2$ ). However, it is surprising to see how the rounds corresponding to large $k$ 's $\left(k=2^{\mu}-1, \mu \geq 3\right)$ lose strength - even with the improperly-argued $\sigma_{k, 1}$ 's in (10). In fact, the third round $(k=7)$ produces no new information for $2^{e}$-torsion lens spaces having $e \geq 2$. Remark 4.12 below gives further details focusing on the situation in [36] for real projective spaces.

The feeding ingredient for the two rounds we need (with $k=1,3$ ) comes from Lemma 4.9 below. Its $e=1$ analogue is proved in [36] by a straightforward application of Milgram's linear algebra techniques in [39]. The general case will be proved in the next section using obstruction theory.

Lemma 4.9. For $\mu=1,2$ and positive integers $\ell$ and $e$, set $i=2^{\mu} \ell-1$, and assume $\ell \geq 2$ when $e>2=\mu$. Then $2^{\mu} \eta_{i, e} \subset \mathbb{R}^{4 i+3}$. If, in addition, $\ell$ is even with $\alpha(\ell) \geq 2$, then $2^{\mu} \eta_{i, e} \subset \mathbb{R}^{4 i+2}$.

Remark 4.10. While the first conclusion in this lemma is directly used within the two inductive rounds referred to above (to form columns 2 and 4 in Table 1), the second 
conclusion is used to get an improvement by one dimension, in certain cases, of the embeddings inductively obtained (to form columns 3 and 5 in Table 1). Note that the label column in Table 1 is considered as the first column.

We now show that Theorem 1.3 is a straightforward consequence of Proposition 4.3 and Lemmas 4.5 and 4.9 .

Proof of Theorem 1.3. Let $(e, n, d)$ be as in the second column of Table 1 , and proceed by induction on $\ell \geq 1$. For the start of the induction, the embedding $L_{3, e} \subset \mathbb{R}^{11}$, apply Proposition 4.3(i) with $k=1, j=1, \alpha=5, \beta=5$ (coming from Remark 2.4), and $\sigma=2$ (as observed in Remark 4.6), using Lemma 4.9 with $\mu=1$ and, of course, $\ell=1$. Then, for the inductive step apply Proposition 4.3(i) with $k=1, j=2 \ell-1$, $\alpha=6, \beta=8 \ell-4$ (one higher than the inductive hypothesis), and $\sigma=\sigma_{1, e}=3$ (as in Lemma 4.5), using Lemma 4.9 with $\mu=1$ and, of course, the current inductive $\ell$.

For $(e, n, d)$ as in the third column of Table 1, apply Proposition 4.3(ii), with $k=1$, $j=2 \ell-1, \alpha=6, \beta=8 \ell-5$ (coming from the first round above), and $\sigma=\sigma_{1, e}=3$ (as in Lemma 4.5), using Lemma 4.9 with $\mu=1$ and $\ell=\frac{n-1}{2}$.

Now let $(e, n, d)$ be as in the fourth column of Table 1 (we only consider the case $e \geq 2$; see Remark 4.11 for a slight strengthening of the method in the original case $e=$ 1 ), and proceed by induction on $\ell \geq 2$. For the inductive step apply Proposition 4.3( $i)$ with $k=3, j=4 \ell-1, \alpha=14, \beta=16 \ell+\delta(e)-15$ (one higher than the inductive hypothesis), and $\sigma=\sigma_{3, e}$ (as in Lemma 4.5), using Lemma 4.9 with $\mu=2$ and, of course, the current inductive $\ell$. This time, in order to ground the induction $(\ell=2)$, we need to show the existence of an embedding

$$
L_{7, e} \subset \mathbb{R}^{17+\delta(e)} \text {. }
$$

For $e \geq 3$, this is given by the second column in Table 1 (with $\ell=3$ ). But for $e=2$, (11) is just the embedding for the special type of triples in the statement of Theorem 1.3.

In order to establish (11) for $e=2$, apply Proposition 4.3(i) with $k=3, j=3$, $\alpha=14, \beta=11$ (coming from the start of the first induction in this proof), and $\sigma=\sigma_{3,2}=5$ (as in Lemma 4.5), using Lemma 4.9 with $\mu=2$ and $\ell=1$, which is still a valid case in Lemma 4.9 (all we need at this point is the weaker embedding $\left.4 \eta_{3,2} \subset \mathbb{R}^{16}\right)$.

Finally, for $(e, n, d)$ as in the fifth column of Table 1, apply Proposition 4.3(ii), with $k=3, j=4 \ell-1, \alpha=14, \beta=16(\ell-1)+\delta(e)$ (coming from the second round above), and $\sigma=\sigma_{3, e}$ (as in Lemma 4.5), using Lemma 4.9 with $\mu=2$ and $\ell=\frac{n-3}{4}$.

Since $\sigma_{1, e}=3$ and the embedding dimension of any $L_{1, e}$ is 5 , the first inductive round (and its improvements) produces embeddings (second and third columns in Table 1) whose Euclidean dimensions are independent of $e$. However, the second round's output (fourth and fifth columns in Table 1) does depend on $e$. The next remark describes the situation (sharpened with the information in [44]) for $e=1$.

Remark 4.11. Rees' PL topological embedding $\mathrm{P}^{7} \subset \mathbb{R}^{10}$ in [44] improves by one dimension the embedding coming from the start of the first inductive round. When this information is fed into the start of the second round, there results an embedding $\mathrm{P}^{15} \subset \mathbb{R}^{25}$, a corresponding improvement of (11) in one dimension (for $e=2$ ), but still 2 dimensions weaker than Rees' PL embedding $P^{15} \subset \mathbb{R}^{23}$ in $[\mathbf{4 4}]$. But when the latter 
embedding is fed into the next step of the second round, there results the (Haefliger smoothable) embedding $P^{23} \subset \mathbb{R}^{39}$, an optimal result according to [8]. Moreover, this situation even shows that Milgram's embedding $4 \eta_{7,1} \subset \mathbb{R}^{31}$ in Lemma 4.9 is optimal, so that the corresponding sharpening in column 5 of Table 1 indeed fails to apply in this case. These two phenomena repeat consistently throughout the second round (for $e=1$ ) yielding the embeddings

$$
\mathrm{P}^{8 j+7} \subset \mathbb{R}^{16 j+7} \text { and } 4 \eta_{4 j-1,1} \subset \mathbb{R}^{16 j-1}, \text { for } j \geq 2
$$

and

$$
\mathrm{P}^{8 j+7} \subset \mathbb{R}^{16 j+6} \text {, for even } j \text { not a power of } 2 .
$$

Note that the first embedding in (12) gives the $e=1$ case of the fourth column in Table 1. This embedding and, therefore, the second embedding in (12) are optimal, according to [8], if $j$ is a power of 2 , that is, when the improvement referred in Remark 4.10 actually fails to apply. Likewise, (13) gives the $e=1$ case of the fifth column in Table 1 . It is worth noticing that, according to [8], the embedding in (13) is currently best known when $j$ is even and $\alpha(j)=2$.

Remark 4.12. Inductive rounds corresponding to values $k=2^{\mu}-1$ with $\mu \geq 3$ have a dramatic loss of strength. In fact, in the case of real projective spaces, this problem (noticed in the paragraph previous to Theorem 1.8 in [36]) led to a rather weak lower bound for the embedding efficiency of $\mathrm{P}^{n}$ - roughly $2 \log _{2}(\alpha(n)$ ), the main (but faulty) theorem in [36]. The reason for this diminished strength of methods comes from the fact that, for $i$ as in Lemma 4.9, the feeding ingredient $2^{\mu} \eta_{i, e} \subset \mathbb{R}^{4 i+3}$ is (in [36]'s wording) "rarely satisfied" when $\mu \geq 3$ (not to mention the embedding in $\mathbb{R}^{4 i+2}$ ), and thus needs to be replaced with a higher dimensional (therefore weaker) Euclidean embedding. We offer here a simple numerical analysis (which requires familiarity with the notation in [36, Lemma 1.5]) of how the problem arises in the case of real projective spaces. The $\ell$-th step in the $\mu$-th inductive round has the form

$$
L_{2^{\mu}-1,1} \subset \mathbb{R}^{\alpha} \text { with } \sigma \text { normal sections, } L_{\ell \cdot 2^{\mu}-1,1} \subset \mathbb{R}^{\beta}, 2^{\mu} \eta_{\ell \cdot 2^{\mu}-1,1} \subset \mathbb{R}^{\sigma+\beta} \Rightarrow \cdots \text {. }
$$

In these conditions, and in order for Proposition 4.3 to have its strongest conclusion, it is necessary that $\sigma+\beta$ be (perhaps one more than) twice the dimension of $L_{\ell \cdot 2^{\mu}-1,1}$. This proves to be the case under the condition " $p \leq \alpha(n)+\kappa(p, n)-\alpha(p+1)$ " of $[\mathbf{3 6}$, Lemma 1.5]. In our terms, such a condition easily translates into

$$
2^{\mu+1}-1 \leq \alpha(\ell)+\mu+\kappa(\mu),
$$

where $\kappa(\mu)=1$ for $\mu=1$, and $\kappa(\mu)=4$ for $\mu \geq 2$. Although (14) always holds when $\mu \leq 2$, it indeed rarely holds for $\mu \geq 3$.

\section{Proof of Lemma 4.9}

We continue to use last section's notation $L_{m, e}$ for the lens space $L^{2 m+1}\left(2^{e}\right)$. Recall that a model for $\tau_{m, e}$, the tangent bundle of $L_{m, e}$, is given by the quotient of the space of pairs $(x, y) \in S^{2 m+1} \times \mathbb{C}^{m+1}$, where $x$ and $y$ are perpendicular, by the diagonal action of $\mathbb{Z} / 2^{e}$. Thus $(x, y)$ and $(\omega x, \omega y)$ are identified in $\tau_{m, e}$ for $\omega \in \mathbb{Z} / 2^{e}$. 
Lemma 5.1. For $m \geq n \geq 0,(m-n) \eta_{n, e}$ is the normal bundle for the embedding $L_{n, e} \subset L_{m, e}$ coming from the first coordinates.

Proof. Recall from the previous section that $(m-n) \eta_{n, e}$ is the quotient space of $S^{2 n+1} \times \mathbb{C}^{m-n}$ by the diagonal action of $\mathbb{Z} / 2^{e}$. Then the map

$$
((x, y),(x, z)) \mapsto(x,(y, z))
$$

produces a linear monomorphism $\tau_{n, e} \oplus(m-n) \eta_{n, e} \hookrightarrow \tau_{m, e}$ over $L_{n, e} \subset L_{m, e}$ that identifies $\tau_{n, e} \oplus(m-n) \eta_{n, e}$ with the restriction of $\tau_{m, e}$ to $L_{n, e}$.

Let $F(t)$ be the Hurwitz-Radon function giving the maximal number of everywhere linearly independent vector fields on $S^{t}$. The following result gives the basis for our obstruction theory approach to Milgram's linear algebra input in [36].

Proposition 5.2. Assume that the restriction to $L_{n, e}$ of the stable normal bundle $\nu: L_{m, e} \rightarrow \mathrm{BO}$ is represented by a d-dimensional bundle classified by the map $\nu^{\prime}$ in the homotopy commutative diagram

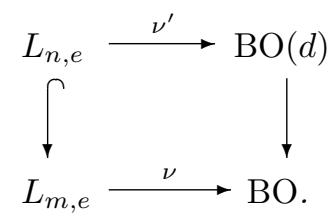

If $2 m+d \geq 4 n+1$ and, in case of equality, $2(m-n) \leq F(2 n+1)$, then there is an embedding $(m-n) \eta_{n, e} \subset \mathbb{R}^{2 m+d+1}$.

Proof. Think of $\nu$ as an $M$-dimensional bundle for $M \gg 0$. After cancelling a few trivial sections, the $L_{n, e}$-restriction of the equality

$$
\tau_{m, e} \oplus \nu=2 m+1+M \text { becomes } \tau_{n, e} \oplus \nu^{\prime \prime}=2 m+d+1,
$$

where $\nu^{\prime \prime}=(m-n) \eta_{n, e} \oplus \nu^{\prime}$. Pick an immersion $L_{n, e} \rightarrow \mathbb{R}^{2 m+d+1}$ with normal bundle $\nu^{\prime \prime}$. If $2 m+d>4 n+1$, this immersion is regularly homotopic to an embedding, an open tubular neighborhood of which can be identified with $\nu^{\prime \prime}$ (producing, in particular, the required embedding for $\left.(m-n) \eta_{n, e}\right)$. The case $2 m+d=4 n+1$ is treated in a similar way, except that the regular deformation argument is replaced by $[\mathbf{3 6}$, Theorem 2.2].

We next show how to reduce Lemma 4.9 to a particular instance of Proposition 5.2. The reader will readily verify that, for $\mu, \ell$, and $e$ as in Lemma 4.9 , the numerical requirements in Proposition 5.2 are satisfied with $n=2^{\mu} \ell-1, m=2^{\mu}(\ell+1)-1$, and $d=2^{\mu+1}(\ell-1)-\lambda$, where

$$
\lambda= \begin{cases}1, & \text { when } \alpha(\ell) \geq 2 \text { and } \ell \equiv 0 \bmod 2 \\ 0, & \text { otherwise. }\end{cases}
$$

Under these conditions, Lemma 4.9 becomes the conclusion of Proposition 5.2. Therefore, settling the existence of a map $\nu^{\prime}$ as in diagram (15) is the only missing task in order to complete the proof of Lemma 4.9. Moreover, since the stable normal bundle of $L_{m, e}$ is well known to be the $-(m+1)$ Whitney multiple of the pull-back of the 
Hopf bundle $H_{m}$ (denoted simply by $H$ if no confusion arises) under the canonical projection $L_{m, e} \rightarrow \mathbb{C P}^{m}$, our real goal becomes showing the existence of the dotted homotopy lifting in the following diagram. We stress that the hypothesis to this aim, namely $\mu, \ell$, and $e$ being as in Lemma 4.9, with $\lambda$ given by (16), will be in force throughout the rest of the section.

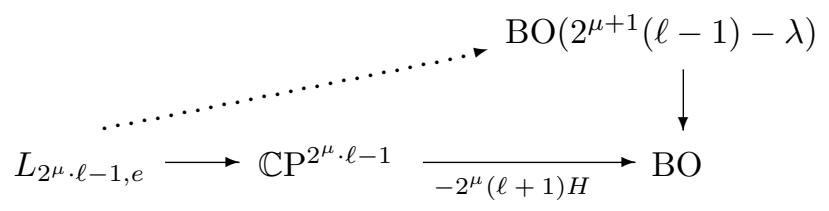

Remark 5.3. The following considerations refer to the case $\lambda=1$ in (16). While the assumed parity of $\ell$ is an important ingredient in the above derivation of Lemma 4.9 from Proposition 5.2, no use is yet made of the condition $\alpha(\ell) \geq 2$. The latter requirement will shortly be identified as the relevant hypothesis for the corresponding (i.e., $\lambda=1$ ) construction of the lifting in (17). In fact, it will be convenient to construct a slightly stronger set of liftings (for $\mu, \ell$, and $e$ ), namely, one where the restriction $\ell \equiv 0 \bmod 2$ is removed from the case $\lambda=1$ of (16).

We next take care of a couple of easy cases of (17).

Case $\ell=1$. (16) gives $\lambda=0$, so we need to show the homotopy triviality of the horizontal composite in (17). This is standard for $e=1$, whereas the case $e \geq 2$ follows from the $\widetilde{K O}$-calculations in [30] (this is the point where we require the hypothesis $e \leq 2$ for $\mu=2$ ). Therefore we assume $\ell \geq 2$ from now on, which will allow us to get the lifting in (17) even at the level of the complex projective space.

Case $\lambda=1$. (The cases with an even $\ell$ correspond to the 'stronger' liftings leading to the improvements referred to in Remark 4.10.) For $\mu=1$, the existence of the required lifting was actually established in $[\mathbf{4 2}]$ provided $\ell \equiv 0 \bmod 2$ - see the proof of Theorem 1 on pages $172-173$ of that paper. We leave for the reader the verification that the required lifting for $\mu=1$ (and any $\ell$ ) follows from an argument similar to the one we now describe for the situation with $\mu=2$. The lifting problem we want to solve is

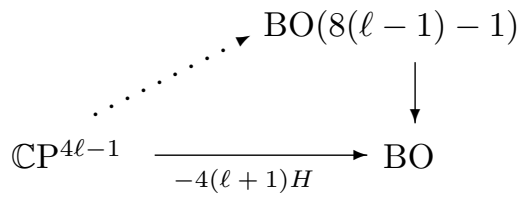

and, interpreted as an upper bound for the geometric dimension of the horizontal map in (18), its solution follows as a consequence of [10, Theorem 1.3(c)]. Indeed, in that result take

$$
\epsilon=3, d=5, \quad m=2 \ell-3, \text { and } p=2^{N}-4(\ell+1),
$$

where $N \gg 0$ (in fact, the summand $2^{N}$ would have to be replaced by any multiple of the order of the Hopf bundle over $\mathbb{C P}^{4 \ell-1}$, but this is immaterial for the 2-primary 
calculations below). Then, with the notation as in (19), the conditions implying the lifting in (18) are:

- $\nu\left(\begin{array}{c}p \\ 2 m+2\end{array}\right) \geq 1$;

- $\nu\left(\begin{array}{c}p \\ 2 m+4\end{array}\right) \geq 3$

- $2 m \geq d-\epsilon$.

Table 1.9 in [10] imposes no further 'additional conditions'. The third inequality is immediate, while the first two are straightforward verifications using the identities

$$
\begin{gathered}
\nu\left(\begin{array}{l}
a \\
b
\end{array}\right)=\alpha(b)+\alpha(a-b)-\alpha(a), \\
\alpha(a-1)=\alpha(a)-1+\nu(a),
\end{gathered}
$$

and

$$
\alpha\left(2^{N}-a\right)=N-\alpha(a-1)
$$

for $N \gg 0$. For instance, the second inequality is verified as follows:

$$
\begin{aligned}
\nu\left(\begin{array}{c}
p \\
2 m+4
\end{array}\right) & =\nu\left(\begin{array}{c}
2^{N}-4(\ell+1) \\
4 \ell-2
\end{array}\right) \\
& =\alpha(4 \ell-2)+\alpha\left(2^{N}-8 \ell-2\right)-\alpha\left(2^{N}-4(\ell+1)\right) \\
& =\alpha(\ell-1)+1+N-\alpha(8 \ell+1)-(N-\alpha(4 \ell+3)) \\
& =\alpha(\ell-1)+1-\alpha(\ell)-1+\alpha(\ell)+2=\alpha(\ell-1)+2 \\
& =\alpha(\ell)-1+\nu(\ell)+2 \geq 2-1+2=3
\end{aligned}
$$

where the inequality in the previous line uses the hypothesis $\alpha(\ell) \geq 2$. In a similar way one checks that $\nu\left(\begin{array}{c}p \\ 2 m+2\end{array}\right)=\alpha(\ell)-1$.

Remark 5.4. Stronger liftings than (18) can be deduced from the Davis-Mahowald technique [10] for certain odd values of $\ell$. For instance, when $\ell=2^{a} u+3$ with odd $u$ and $a \geq 2$, we get a lifting to $\mathrm{BO}(8(\ell-1)-2)$ if $u=1$, and to $\mathrm{BO}(8(\ell-1)-3)$ if $u>1$. These follow from $[\mathbf{1 0}$, Theorem $1.3(\mathrm{c})]$ by replacing $\epsilon=3$ in (19) with $\epsilon=2$ and $\epsilon=1$, respectively.

It remains to consider the lifting (17) for $\ell \geq 2$ and $\lambda=0$. According to (16), as modified in Remark 5.3, this means in fact

$$
\ell=2^{\varrho} \text {, with } \varrho>0 \text {. }
$$

For this, we first observe that the horizontal composite in (17) factors through the corresponding quaternionic projective space as

$$
L_{2^{\mu} \ell-1, e} \rightarrow \mathbb{C P}^{2^{\mu} \ell-1} \rightarrow \mathbb{H} \mathrm{P}^{2^{\mu-1} \ell-1} \rightarrow \mathrm{BO}
$$

(see [46, Lemma 5.4]). Since the case with $\mu=2$ is far more complicated, we first dispose of the (rather elementary) situation for $\mu=1$. The point is that the fiber of $\mathrm{BO}(4 \ell-4) \rightarrow \mathrm{BO}$ is $(4 \ell-5)$-connected so that, at the level of the quaternionic projective space, the obstructions for the required lifting lie in trivial groups. 
We have saved the most interesting case for last, namely, the one having $\lambda=0$ and $\mu=2$, with $\ell$ as in (20). Thus, the proof of Lemma 4.9 will be complete once we solve the instance of (17) summarized by the following result (where we have set $j=\ell-1$, an odd number in view of (20)).

Proposition 5.5. The homotopy lifting problem

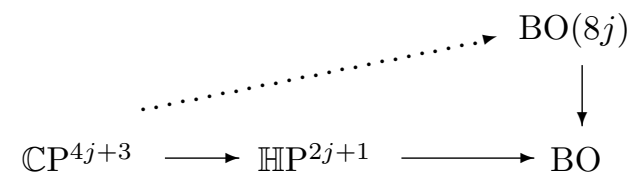

can be solved for any positive odd ${ }^{5}$ integer $j$, where the horizontal composite classifies the realification of the Whitney multiple $-4(j+2) H_{4 j+3}$.

We have already said that the instance of (17) represented by Proposition 5.5 (as well as the previous instances of (17) we have already solved) is proved in [36] for real projective spaces by means of direct linear algebra constructions. The lack of such a technique for higher torsion lens spaces led us to analyze alternative approaches to Proposition 5.5 that could not only explain in a simple form the corresponding $e=1$ instance in (17), but that would allow us to obtain a suitable generalization to higher torsion lens spaces. The tool that proved to simultaneously solve these problems is Mahowald's theory of modified Postnikov towers (MPT's) [34], as refined in [14], which is freely used from this point on. Indeed, such an analysis is actually very simple in the case of real projective spaces as the whole lifting can be sorted out through easy primary indeterminacy arguments. This gave a first indication of the viability of the method in the general case. But its complete success comes from a careful secondary indeterminacy analysis - the heart of the proof below - that leads to the required lifting (17) for every torsion lens space (in the form of Proposition 5.5).

The remainder of this section is devoted to the construction of the dotted homotopy lifting in Proposition 5.5. The proof is a bit lengthy, so we divide it into three main steps. The first two work for any (even or odd) $j$; the hypothesis of having an odd $j$ will be applied only near the end of the third step, in order to evaluate a certain nontrivial secondary cohomology operation.

Step 1. Description of the MPT we use. Since $-4(j+2) H_{4 j+3}$ has trivial first and second Stiefel-Whitney classes, the classifying map of this bundle can be further factored through $B$ Spin. We denote the maps in the resulting factorization as

$$
\mathbb{C P}^{4 j+3} \stackrel{f}{\rightarrow} \mathbb{H P}^{2 j+1} \stackrel{g}{\rightarrow} B \mathrm{Spin} \stackrel{h}{\rightarrow} \mathrm{BO},
$$

and note that it suffices to lift the composite $g f$ to $B \operatorname{Spin}(8 j)$. In the range under consideration the fiber of $B \operatorname{Spin}(8 j) \rightarrow B$ Spin is the stunted real projective space $\mathrm{P}_{8 j}^{8 j+6}$ - the quotient of $\mathrm{P}^{8 j+6}$ with $\mathrm{P}^{8 j-1}$ collapsed to a point. This is the Thom space

\footnotetext{
${ }^{5}$ Remark 5.4 indicates stronger liftings for an even $j$. In fact, the case $\lambda=1$ discussed in (18) gives a lifting one higher when $\alpha(j+1) \geq 2$. On the other hand, the final remarks in this section explain why Proposition 5.5 gives an optimal result in the remaining case $j=2^{\varrho}-1, \varrho>0$.
} 
of the bundle $8 j \xi_{6}$ (notation as in Section 2), which is a stable coreducible complex by standard $K$-theory considerations. Thus $\mathrm{P}_{8 j}^{8 j+6}$ splits as $S^{8 j} \vee \mathrm{P}_{8 j+1}^{8 j+6}$, with homotopy groups through dimension $8 j+5$ given in the Adams chart (see [35, Tables 8.1, 8.2, and 8.9])

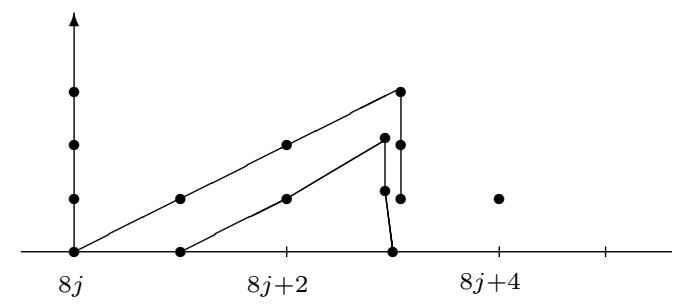

where any potentially nontrivial Adams differential is ruled out by the splitting. The condition $j \geq 1$ assures that we are in the range for a $(8 j+6)$-MPT to exist; it takes the form

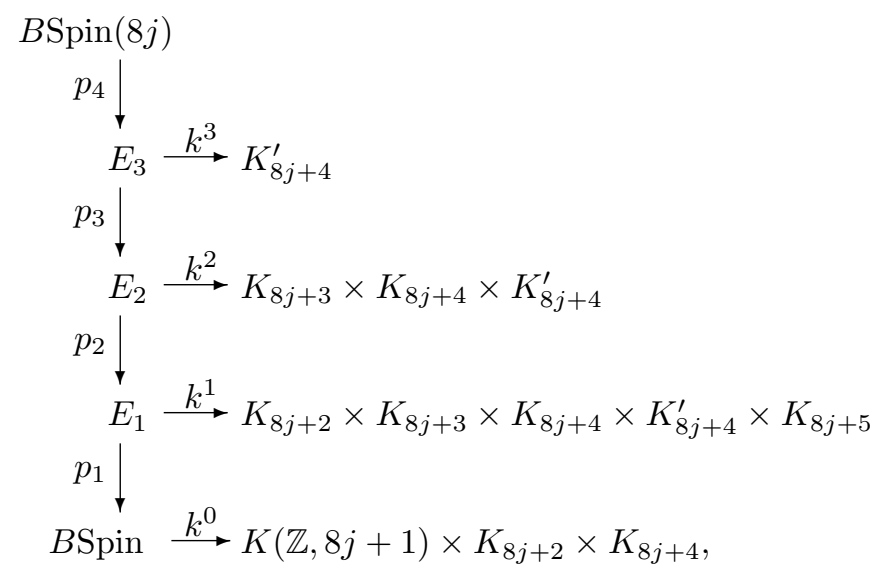

where $K_{m}$ stands for the Eilenberg-MacLane space $K(\mathbb{Z} / 2, m)$. We let $K(i)$ and $\mu_{i}: K(i) \times E_{i} \rightarrow E_{i}$ stand for the fiber and the action, respectively, in the principal fibration $p_{i}$. To conclude the MPT setting, we remark that a standard calculation gives the following characterizations for the $k$-invariants:

$$
\begin{aligned}
& \mu_{1}^{*}\left(k_{8 j+2}^{1}\right)=1 \otimes k_{8 j+2}^{1}+\mathrm{Sq}^{2} \iota_{8 j} \otimes 1, \\
& \mu_{1}^{*}\left(k_{8 j+3}^{1}\right)=1 \otimes k_{8 j+3}^{1}+\mathrm{Sq}^{2} \iota_{8 j+1} \otimes 1, \\
& \mu_{1}^{*}\left(k_{8 j+4}^{1}\right)=1 \otimes k_{8 j+4}^{1}+\mathrm{Sq}^{1} \iota_{8 j+3} \otimes 1+\mathrm{Sq}^{2} \mathrm{Sq}^{1} \iota_{8 j+1} \otimes 1, \\
& \mu_{1}^{*}\left(k_{8 j+4}^{\prime 1}\right)=1 \otimes k_{8 j+4}^{\prime 1}+\mathrm{Sq}^{4} \iota_{8 j} \otimes 1+\iota_{8 j} \otimes w_{4}, \\
& \mu_{1}^{*}\left(k_{8 j+5}^{1}\right)=1 \otimes k_{8 j+5}^{1}+\mathrm{Sq}^{4} \iota_{8 j+1} \otimes 1+\iota_{8 j+1} \otimes w_{4}, \\
& \mu_{2}^{*}\left(k_{8 j+3}^{2}\right)=1 \otimes k_{8 j+3}^{2}+\mathrm{Sq}^{2} \iota_{8 j+1} \otimes 1, \\
& \mu_{2}^{*}\left(k_{8 j+4}^{2}\right)=1 \otimes k_{8 j+4}^{2}+\mathrm{Sq}^{1} \iota_{8 j+3} \otimes 1+\mathrm{Sq}^{2} \iota_{8 j+2} \otimes 1, \\
& \mu_{2}^{*}\left(k_{8 j+4}^{\prime 2}\right)=1 \otimes k_{8 j+4}^{\prime 2}+\mathrm{Sq}^{1} \iota_{8 j+3}^{\prime} \otimes 1+\mathrm{Sq}^{2} \mathrm{Sq}^{1} \iota_{8 j+1} \otimes 1, \\
& \mu_{3}^{*}\left(k_{8 j+4}^{\prime 3}\right)=1 \otimes k_{8 j+4}^{\prime 3}+\mathrm{Sq}^{1} \iota_{8 j+3}^{\prime} \otimes 1+\mathrm{Sq}^{2} \iota_{8 j+2} \otimes 1 .
\end{aligned}
$$


Here $\iota_{t}$ stands for the relevant fundamental class in the various fibers $K(i)$ (note that we have systematically primed $k$-invariants, Eilenberg-Mac Lane spaces, and fundamental classes coming from the three classes in the right-hand tower of height 3 in the chart above).

Step 2. Basic (primary) MPT analysis. Since the binomial coefficient $\left(\begin{array}{c}-2 j-4 \\ 2 j+1\end{array}\right)$ is even, $k_{8 j+4}^{0}$ is trivial over $\mathbb{H P}^{2 j+1}$; the other two $k^{0}$-invariants are also trivial over $\mathbb{H P}^{2 j+1}$ by dimensional reasons. Therefore $g$ lifts in $(22)$ to a map $\ell_{1}: \mathbb{H P}^{2 j+1} \rightarrow E_{1}$ whose only possibly nontrivial $k^{1}$-invariants are $k_{8 j+4}^{1}$ and $k_{8 j+4}^{\prime 1}$. We now show that these two $k^{1}$-invariants map trivially.

To deal with $\ell_{1}^{*}\left(k_{8 j+4}^{\prime 1}\right)$ we first note that extending the Adams chart through dimension $8 j+7$ extends the $(8 j+6)-$ MPT to an $(8 j+8)-$ MPT with only one extra $k^{0}$-invariant, $k_{8 j+8}^{0}$. Since $\left(\begin{array}{c}-2 j-4 \\ 2 j+2\end{array}\right)$ is even, $k_{8 j+8}^{0}$ is trivial over $\mathbb{H P}^{2 j+2}$ and, thus, the lifting $\ell_{1}: \mathbb{H} \mathrm{P}^{2 j+1} \rightarrow E_{1}$ extends to a lifting $\mathbb{H} \mathrm{P}^{2 j+2} \rightarrow E_{1}$. In this extended MPT, there is a $k^{2}$-invariant, $k_{8 j+7}^{2}$, defined by the relation

$$
\left(\mathrm{Sq}^{4}+w_{4}\right) k_{8 j+4}^{\prime 1}+\left(\mathrm{Sq}^{6}+w_{6}\right) k_{8 j+2}^{1}=0
$$

in $E_{1}$. This implies $\left(\mathrm{Sq}^{4}+w_{4}\right) \ell_{1}^{*}\left(k_{8 j+4}^{\prime 1}\right)=0$. Thus, assuming $\ell_{1}^{*}\left(k_{8 j+4}^{\prime 1}\right)$ is the nontrivial class in $H^{8 j+4}\left(\mathbb{H P}^{2 j+2} ; \mathbb{Z} / 2\right)$ yields a contradiction since $\mathrm{Sq}^{4}$ of this class is nontrivial, but $w_{4}=0$ for $g$.

To deal with $\ell_{1}^{*}\left(k_{8 j+4}^{1}\right)$ we need the fact that $g$ lifts to $B \operatorname{Spin}(8 j+1)$. This follows from a straightforward application of $[\mathbf{9}$, Theorem $1.3(\mathrm{~b})]$ - in a similar way to our argument for (18), but this time with quaternions instead of complex numbers. With this information at hand, we consider the following diagram of MPT's, where the lefthand tower is (22), the right-hand tower is the $\mathrm{MPT}$ for $B \operatorname{Spin}(8 j+1) \rightarrow B \operatorname{Spin}$, and the map of MPT's is induced by the canonical map $j: B \operatorname{Spin}(8 j) \rightarrow B \operatorname{Spin}(8 j+1)$ :

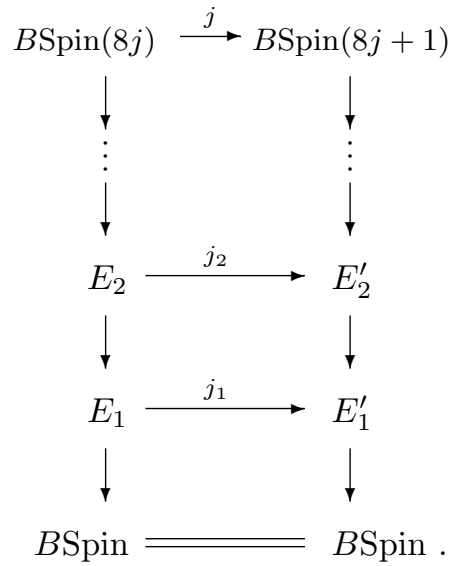

Moreover, the effect in homotopy (up to dimension $8 j+3$ ) of the mapping between fibers can be read in the Adams charts (see [35, Tables 8.1-8.2 for even $j$, and 8.9-8.10 
for odd $j]$ )

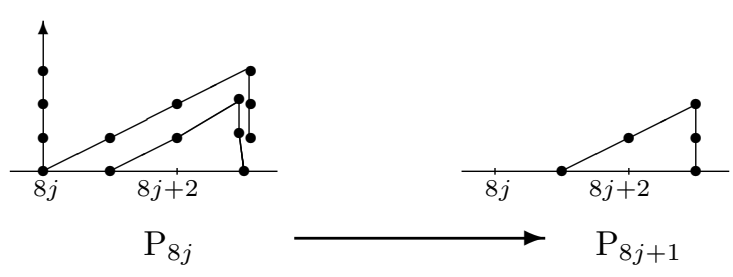

In particular, the $(8 j+4)$-dimensional $k^{1}$-invariant on the right-hand side in $(32)$ maps into $k_{8 j+4}^{1}$ in $(22)$. Now, since $g$ lifts to $B \operatorname{Spin}(8 j+1)$ the composite $j_{1} \ell_{1}$ has trivial $k^{1}$-invariants (with trivial indeterminacies). It follows that $\ell_{1}^{*}\left(k_{8 j+4}^{1}\right)=0$.

We have proved:

Lemma 5.6. The map $g$ lifts in (22) to a map $\ell_{2}$ : $\mathbb{H} \mathrm{P}^{2 j+1} \rightarrow E_{2}$.

Remark 5.7. Let $\tilde{\ell}_{2}=\ell_{2} f$. Note that $\tilde{\ell}_{2}^{*}\left(k_{8 j+3}^{2}\right)$ is trivial by dimensional reasons, whereas (29) and the fact that $\mathrm{Sq}^{2}$ acts nontrivially on $H^{8 j+2}\left(\mathbb{C P}^{4 j+3} ; \mathbb{Z} / 2\right)$ imply we can kill $\widetilde{\ell}_{2}^{*}\left(k_{8 j+4}^{2}\right)$ by primary indeterminacy. Moreover, (30) shows that this killing of $\widetilde{\ell}_{2}^{*}\left(k_{8 j+4}^{2}\right)$ does not modify $\widetilde{\ell}_{2}^{*}\left(k_{8 j+4}^{\prime 2}\right)$. Also, $(31)$ shows that the $k^{3}$-invariant of any possible lifting $\widetilde{\ell}_{3}: \mathbb{C P}^{4 j+3} \rightarrow E_{3}$ of $g f$ can be killed by primary indeterminacy.

Step 3. Secondary indeterminacy. We now prove the next result, which coupled with Remark 5.7 implies Theorem 5.5.

Proposition 5.8. There is a lifting $\widetilde{\ell}_{2}^{\prime}: \mathbb{C P}^{4 j+3} \rightarrow E_{2}$ of $g f$ in (22) that has trivial $k_{8 j+4}^{\prime 2}$-invariant.

Proof. We show that a nontrivial $\widetilde{\ell}_{2}^{*}\left(k_{8 j+4}^{\prime 2}\right)$ can be corrected through secondary indeterminacy. To this end, we first need to make a slight adjustment in our MPT: We already noticed that $w_{4}=0$ for $g$, so that this map factors as

$$
\mathbb{H} \mathrm{P}^{2 j+1} \stackrel{g^{\prime}}{\rightarrow} F \rightarrow B \text { Spin, }
$$

where $F$ is the fiber of $w_{4}: B$ Spin $\rightarrow K_{4}$. Note that, since $\pi_{4}(B$ Spin $)=\mathbb{Z}, F$ is still 3 -connected (not 4-connected), but we have killed $w_{4}$ in $F$. We will prove the required condition for $g^{\prime} f$ with (22) replaced by the induced MPT over $F$; therefore we extend accordingly our notation for (22) to this MPT over $F$. In particular, Remark 5.7 gives us a corresponding lifting

$$
\widetilde{\ell}_{2}: \mathbb{C P}^{4 j+3} \rightarrow E_{2}
$$

of $g^{\prime} f$, and we will show how to alter (if needed) its $k_{8 j+4}^{\prime 2}$-invariant by secondary indeterminacy coming from the class $y^{4 j} \in H^{8 j}\left(\mathbb{C P}^{4 j+3} ; \mathbb{Z} / 2\right)$, where $y$ stands for the generator in $H^{2}\left(\mathbb{C} P^{4 j+3} ; \mathbb{Z} / 2\right)$. Although-although not needed, we remark that this is in fact the only way to correct the problematic $k_{8 j+4}^{\prime 2}$-invariant. The next considerations are preparatory.

We start by expanding the MPT over $F$ (up to stage $E_{2}$ ) to the commutative 
diagram

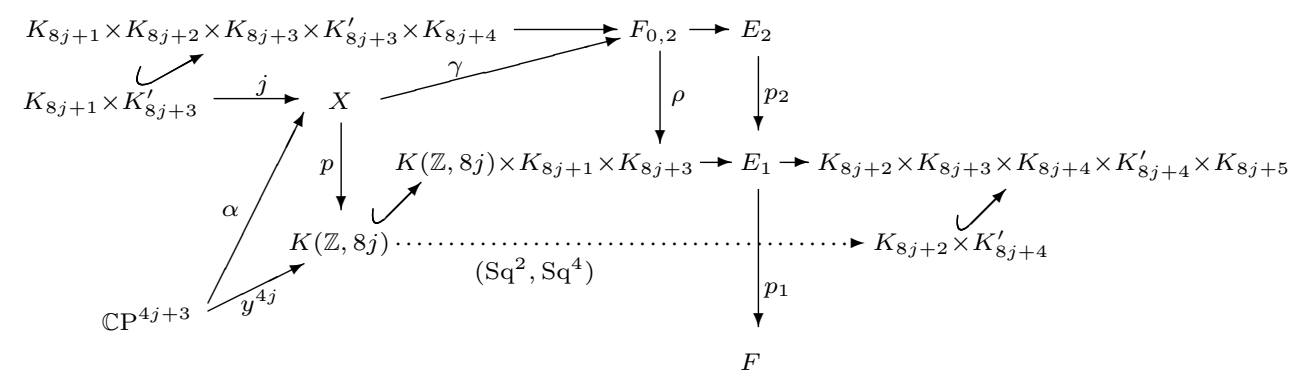

Here the square involving $\rho$ and $p_{2}$ is a pull-back, so that $F_{0,2}$ can be thought of as the common fiber of (a) the composite $p_{1} p_{2}$, and (b) the middle horizontal composite

$$
K(\mathbb{Z}, 8 j) \times K_{8 j+1} \times K_{8 j+3} \rightarrow E_{1} \rightarrow K_{8 j+2} \times K_{8 j+3} \times K_{8 j+4} \times K_{8 j+4}^{\prime} \times K_{8 j+5} .
$$

Likewise, $p: X \rightarrow K(\mathbb{Z}, 8 j)$ is the fiber inclusion of the dotted map $\left(\mathrm{Sq}^{2}, \mathrm{Sq}^{4}\right)$. The map $\gamma: X \rightarrow F_{0,2}$ exists in view of the commutativity of the square involving the $\left(\mathrm{Sq}^{2}, \mathrm{Sq}^{4}\right)$ map (see $\left.(23)-(27)\right)$. The indicated lifting $\alpha$ of $y^{4 j}$ exists since

$$
\left(\mathrm{Sq}^{2}, \mathrm{Sq}^{4}\right)\left(y^{4 j}\right)=0 \text {. }
$$

Next we describe suitable variations of the principal actions for $p_{1}$ and $p_{2}$ in the MPT over $F$. Recall $K(1)$ and $K(2)$ denote, respectively, $K(\mathbb{Z}, 8 j) \times K_{8 j+1} \times K_{8 j+3}$ and $K_{8 j+1} \times K_{8 j+2} \times K_{8 j+3} \times K_{8 j+3}^{\prime} \times K_{8 j+4}$. We define $\mu_{1}^{\prime}: F_{0,2} \times E_{1} \rightarrow E_{1}$ to be the composite

$$
F_{0,2} \times E_{1} \stackrel{\rho \times 1}{\longrightarrow} K(1) \times E_{1} \stackrel{\mu_{1}}{\longrightarrow} E_{1} .
$$

We then have the diagram

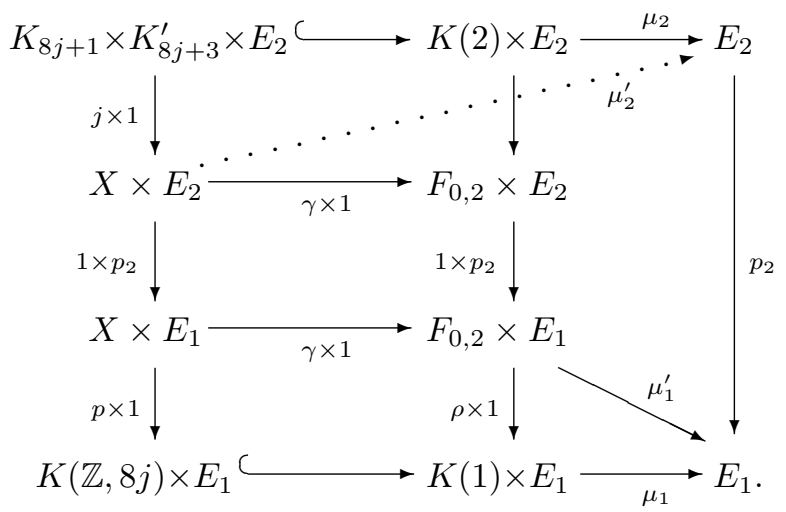

The dotted map $\mu_{2}^{\prime}: X \times E_{2} \longrightarrow E_{2}$, rendering a commutative diagram, exists since the composite

$$
X \times E_{2} \stackrel{\gamma \times p_{2}}{\longrightarrow} F_{0,2} \times E_{1} \stackrel{\mu_{1}^{\prime}}{\longrightarrow} E_{1} \stackrel{k^{1}}{\longrightarrow} K_{8 j+2} \times K_{8 j+3} \times K_{8 j+4} \times K_{8 j+4}^{\prime} \times K_{8 j+5}
$$

is null-homotopic as shown by the following chase of classes (where the symbol "ฯ" 
stands for the map induced by the bottom inclusion in the diagram above):

$$
\begin{aligned}
& k_{8 j+2}^{1} \stackrel{\mu_{1}}{\longrightarrow} 1 \otimes k_{8 j+2}^{1}+\mathrm{Sq}^{2} \iota_{8 j} \otimes 1 \hookrightarrow 1 \otimes k_{8 j+2}^{1}+\mathrm{Sq}^{2} \iota_{8 j} \otimes 1 \stackrel{p \times 1}{\longrightarrow} 1 \otimes k_{8 j+2}^{1} \stackrel{1 \times p_{2}}{\longrightarrow} 0, \\
& k_{8 j+3}^{1} \stackrel{\mu_{1}}{\longrightarrow} 1 \otimes k_{8 j+3}^{1}+\mathrm{Sq}^{2} \iota_{8 j+1} \otimes 1 \hookrightarrow 1 \otimes k_{8 j+3}^{1} \stackrel{p \times 1}{\longrightarrow} 1 \otimes k_{8 j+3}^{1} \stackrel{1 \times p_{2}}{\longrightarrow} 0, \\
& k_{8 j+4}^{1} \stackrel{\mu_{1}}{\longrightarrow} 1 \otimes k_{8 j+4}^{1}+\mathrm{Sq}^{1} \iota_{8 j+3} \otimes 1+\mathrm{Sq}^{2} \mathrm{Sq}^{1} \iota_{8 j+1} \otimes 1 \hookrightarrow 1 \otimes k_{8 j+4}^{1} \stackrel{p \times 1}{\longrightarrow} 1 \otimes k_{8 j+4}^{1} \stackrel{1 \times p_{2}}{\longrightarrow} 0, \\
& k_{8 j+4}^{\prime 1} \stackrel{\mu_{1}}{\longrightarrow} 1 \otimes k_{8 j+4}^{\prime 1}+\mathrm{Sq}^{4} \iota_{8 j} \otimes 1 \hookrightarrow 1 \otimes k_{8 j+4}^{\prime 1}+\mathrm{Sq}^{4} \iota_{8 j} \otimes 1 \stackrel{p \times 1}{\longrightarrow} 1 \otimes k_{8 j+4}^{\prime 1} \stackrel{1 \times p_{2}}{\longrightarrow} 0, \\
& k_{8 j+5}^{1} \stackrel{\mu_{1}}{\longrightarrow} 1 \otimes k_{8 j+5}^{1}+\mathrm{Sq}^{4} \iota_{8 j+1} \otimes 1 \hookrightarrow 1 \otimes k_{8 j+5}^{1} \stackrel{p \times 1}{\longrightarrow} 1 \otimes k_{8 j+5}^{1} \stackrel{1 \times p_{2}}{\longrightarrow} 0 .
\end{aligned}
$$

Note that, in the last two rows, summands with a $w_{4}$ are trivial by construction of $F$. (For readers familiar with [47]: the point of this explicit calculation is to make sure that all terms in the chase lie in wedge portions of the relevant spaces, a point argued in $[\mathbf{4 7}]$ just from easy dimensional reasons.)

We are finally in a position to explicitly indicate how to kill (if needed), through secondary indeterminacy, a nontrivial $k_{8 j+4}^{\prime 2}$-invariant for (33). Start with the composite

$$
\widetilde{\ell}_{1}^{\prime}=\mu_{1}^{\prime}\left((\gamma \alpha) \times\left(p_{2} \widetilde{\ell}_{2}\right)\right): \mathbb{C} \mathrm{P}^{4 j+3} \rightarrow E_{1},
$$

the (primary) modification of $\widetilde{\ell}_{1}=p_{2} \widetilde{\ell}_{2}$ by the class $y^{4 j}$. From the diagram that defines $\mu_{2}^{\prime}$ we have, first, that an explicit lifting of $\widetilde{\ell}_{1}^{\prime}$ is given by the map

$$
\widetilde{\ell}_{2}^{\prime}=\mu_{2}^{\prime}\left(\alpha \times \widetilde{\ell}_{2}\right): \mathbb{C} P^{4 j+3} \rightarrow E_{2}
$$

and, second, that equation (30) translates, by dimensional reasons, into

$$
\left(\mu_{2}^{\prime}\right)^{*}\left(k_{8 j+4}^{\prime 2}\right)=1 \otimes k_{8 j+4}^{\prime 2}+a \otimes 1+\iota_{8 j} \otimes b,
$$

where $a \in H^{8 j+4}(X ; \mathbb{Z} / 2)$ and $b \in H^{4}\left(E_{2} ; \mathbb{Z} / 2\right)$, and where the former element satisfies

$$
j^{*}(a)=\mathrm{Sq}^{1} \iota_{8 j+3}^{\prime} \otimes 1+\mathrm{Sq}^{2} \mathrm{Sq}^{1} \iota_{8 j+1} \otimes 1 .
$$

In particular, the $k_{8 j+4}^{\prime 2}$-invariant of $\widetilde{\ell}_{2}^{\prime}$ is computed as

$$
\begin{aligned}
\left(\widetilde{\ell}_{2}^{\prime}\right)^{*}\left(k_{8 j+4}^{\prime 2}\right) & =\left(\alpha \times \widetilde{\ell}_{2}\right)^{*}\left(1 \otimes k_{8 j+4}^{\prime 2}+a \otimes 1+\iota_{8 j} \otimes b\right) \\
& =\widetilde{\ell}_{2}^{*}\left(k_{8 j+4}^{\prime 2}\right)+\alpha^{*}(a)+\alpha^{*}\left(\iota_{8 j}\right) \cdot \widetilde{\ell}_{2}^{*}(b) .
\end{aligned}
$$

Therefore we will be done once we establish the two relations

$$
\alpha^{*}(a)=y^{4 j+2} \text { and } \tilde{\ell}_{2}^{*}(b)=0 .
$$

By construction $\alpha^{*}(a) \in \Phi\left(y^{4 j}\right)$, where $\Phi$ is the secondary operation determined by the class $a \in H^{8 j+4}(X ; \mathbb{Z} / 2)$ (subject to (34)) in the diagram

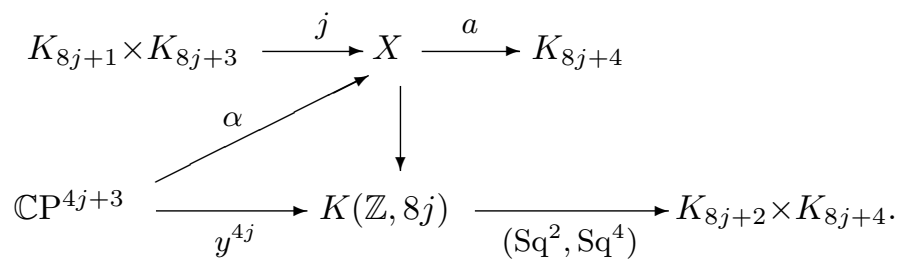


This operation is associated to the relation $\left(\mathrm{Sq}^{2} \mathrm{Sq}^{1}\right) \cdot \mathrm{Sq}^{2}+\mathrm{Sq}^{1} \cdot \mathrm{Sq}^{4}=0$ (for our purposes this is a relation on elements coming from integral classes, so that $\mathrm{Sq}^{1}$ acts trivially on them). But in [17, Theorem A] it is shown that $\Phi\left(y^{4 j}\right)=y^{4 j+2}$ for odd $j$ (with trivial indeterminacy), giving the first equality in (35). The second equality in (35) is much simpler. Assume $b \neq 0$ and let $P_{1}$ stand for the first Pontryagin class of the universal vector bundle over $B$ Spin. Since $H^{4}(B$ Spin; $\mathbb{Z})=\mathbb{Z}$ is generated by $P_{1} / 2$, it follows that $H^{4}(F ; \mathbb{Z})=\mathbb{Z}$ is generated by $P_{1}$. Then the mod 2 reduction of $P_{1}$ must correspond to $b$ under

$$
\mathbb{Z} / 2=H^{4}(F ; \mathbb{Z} / 2) \stackrel{p_{2}^{*}}{\approx} H^{4}\left(E_{1} ; \mathbb{Z} / 2\right) \stackrel{p_{2}^{*}}{\approx} H^{4}\left(E_{2} ; \mathbb{Z} / 2\right) .
$$

Thus $\widetilde{\ell_{2}^{*}}(b)$ is the first Pontryagin class of $-4(j+2) H$, which equals

$$
(-4(j+2)) y^{2}=0
$$

We close this section with a final word about the optimality of the obstruction theory methods. As we have seen, the basis for Lemma 4.9 comes (rather indirectly) from the existence of diagrams of the form (15), settled here by means of modified Postnikov towers - and by direct linear algebra constructions in [36]. Of course, the smaller $d$ one can use in (15), the better are the results produced by the inductive rounds in Section 4. Now, in Remark 4.11 we observed the optimality of some of the embeddings in the conclusion of Lemma 4.9 for $e=1$. In particular, we get the optimality of the corresponding $d$ used in (15). The point we want to stress here is that the latter optimality holds for any $e$, in view of the compatibility of the embeddings $L_{n, e} \hookrightarrow L_{m, e}$ under the canonical projections $L_{t, e} \rightarrow L_{t, e+1}$. The net outcome to remark is that the homotopy lifting in Proposition 5.5 turns out to be optimal when $j$ is one less than a power of 2 -the critical $\mu=2$ case in (20). Together with the corresponding embeddings of lens space, this infinite family of optimal liftings justifies the several pages of MPT manipulations.

\section{Immersion vs. embedding dimension}

In this final section of the paper we focus on certain subtle points arising when comparing the behavior of the (immersion and) embedding dimensions of $2^{e}$-torsion lens spaces, as $e$ varies. The case $t=2^{e}$ of the following easy observation was mentioned in $[\mathbf{1 8}]$ as a convenient way to take advantage of known immersion results for (real and complex) projective spaces when studying the immersion problem for lens spaces.

Fact 6.1. Any codimension- $k$ Euclidean immersion for $\mathbb{C P}^{n}$ brings for free a codimension- $k$ Euclidean immersion for any $t$-torsion lens space $L^{2 n+1}(t)$. Likewise, when $t^{\prime}$ divides $t$, any codimension- $k$ Euclidean immersion of $L^{2 n+1}(t)$ brings for free a codimension- $k$ Euclidean immersion of $L^{2 n+1}\left(t^{\prime}\right)$.

Unfortunately, the first (and potentially the second) statement(s) in Fact 6.1 readily fails when "immersion" is replaced by "embedding". For instance, $\mathbb{C P}^{1}$ embeds in $\mathbb{R}^{3}$, but as observed in Remark 2.4, no 3-dimensional lens space embeds in $\mathbb{R}^{4}$. After discussing how this problem arises and how it could be mended, we deal, in Remark 6.4 below, with the potential usefulness of such a possibly mended embedding analogue. 
For the time being we note that this initial problem could just as well be a facet of the following very peculiar situation:

Fact 6.2. With the exception of $S^{1}$ and $\mathrm{P}^{7}$, which are well known to be parallelizable manifolds, 3-dimensional lens spaces are the only parallelizable manifolds among lens spaces. In more detail, Lemma 3.2 and Proposition 6.3 (below) give the parallelizability of all 3-dimensional lens spaces (the same argument gives the well-known parallelizability of any compact orientable 3-dimensional manifold), whereas it is proved in [32] that, except for $\mathrm{P}^{7}$ and $L^{2 p-1}(p)$ for $p$ an odd prime (the latter is stably parallelizable, but not parallelizable), no other lens space of dimension $\geq 5$ is stably parallelizable.

Proposition 6.3. Any 3-dimensional spin bundle over a 3-dimensional complex is trivial.

Proof. This follows from the fact that $B \operatorname{Spin}(3)=B S^{3}$ is 3 -connected.

Here is an explicit way of looking (within Haefliger's metastable range) at the problems arising in the quest for an embedding analogue of the second statement of Fact 6.1. Consider the configuration space $F_{\mathbb{Z} / 2^{e}}\left(S^{2 n+1}, 2\right)$ consisting of pairs in $S^{2 n+1} \times S^{2 n+1}$ generating different $\mathbb{Z} / 2^{e}$ orbits. As observed in [20, Lemma 5.1], for $k \geq 3(n+1)$, Haefliger's characterization [22, Théorème $\left.1^{\prime}\right]$ for the existence of an embedding $L^{2 n+1}\left(2^{e}\right) \subset R^{k}$ decodes into the existence of a map $\alpha: F_{\mathbb{Z} / 2^{e}}\left(S^{2 n+1}, 2\right) \rightarrow$ $S^{k-1}$ satisfying

$$
\alpha(\omega x, y)=\alpha(x, y)=\alpha(x, \omega y) \text { and } \alpha(x, y)=-\alpha(y, x)
$$

for $(x, y) \in F_{\mathbb{Z} / 2^{e}}\left(S^{2 n+1}, 2\right)$, and $\omega \in \mathbb{Z} / 2^{e}$. Now, although any map satisfying (36) for all $\omega \in \mathbb{Z} / 2^{e}$ will certainly satisfy the same requirements for all $\omega \in \mathbb{Z} / 2^{e-1}$, the strict inclusion $F_{\mathbb{Z} / 2^{e}}\left(S^{2 n+1}, 2\right) \subset F_{\mathbb{Z} / 2^{e-1}}\left(S^{2 n+1}, 2\right)$ can only be used to interpret, as an extension problem, the embedding analogue of the second statement in Fact 6.1.

Despite the above problems, it might still be the case that a restricted embedding version of Fact 6.1 holds. The following considerations are meant to shed evidence on such a possibility. To this end, it will be convenient to use the notation $\operatorname{Emb}(M)$ for the smallest dimension of the Euclidean space where the given manifold $M$ can be embedded. The information we use below about these numbers is taken from [8], in the case of real projective spaces, and from $[\mathbf{3}, \mathbf{4 1}]$, in the case of complex projective spaces.

First of all, the considerations in Fact 6.2 indicate that, in asking whether an embedding analogue of Fact 6.1 holds, it might be fair to exclude the case $n=1$. In fact, we should exclude the whole family $\alpha(n)=1$ as, in such a case, $\operatorname{Emb}\left(\mathbb{C P}^{n}\right)=4 n-1$, but according to Theorem 1.2, $\operatorname{Emb}\left(L^{2 n+1}\left(2^{e}\right)\right)=4 n+1$ for all $e \geq 1$ - one dimension higher than what the embedding analogue of Fact 6.1 would anticipate.

Assuming now $\alpha(n)>1$, there does not seem to be an immediate problem, at least at the outset (but perhaps mainly due to a lack of information), for a potential embedding analogue of Fact 6.1. For instance, when $n=6$ (the first such case with even $n), \operatorname{Emb}\left(\mathbb{C P}^{6}\right) \in\{21,22\}$ whereas $\operatorname{Emb}\left(\mathrm{P}^{13}\right) \in\{22,23\}$-three out of the four possibilities being compatible with a potential embedding analogue of Fact 6.1. 
But for odd $n$ the situation is much nicer, and the problem discussed above for $\alpha(n)=1$ is replaced by the following families ${ }^{6}$ that are fully compatible with a possible embedding version of Fact 6.1. Indeed:

(a) For $n=2^{e}+1 \geq 3, \operatorname{Emb}\left(\mathbb{C P}^{n}\right)=2^{e+2}+1$, whereas $\operatorname{Emb}\left(\mathrm{P}^{2 n+1}\right) \leq 2^{e+2}+2$ if $e \geq 2$.

(b) For $n=2^{e}+3 \geq 7, \quad \operatorname{Emb}\left(\mathbb{C P}^{n}\right) \in 2^{e+2}+\{7,8\}$, whereas $\operatorname{Emb}\left(\mathrm{P}^{2 n+1}\right)=$ $2^{e+2}+7$ if $e \geq 3$.

(c) For $n=2^{e}+5 \geq 13$, both $\operatorname{Emb}\left(\mathbb{C P}^{n}\right)$ and $\operatorname{Emb}\left(\mathrm{P}^{2 n+1}\right)$ are of the form $2^{e+2}+\{15,16\}$.

(d) For $n=2^{e}+7 \geq 15, \operatorname{Emb}\left(\mathbb{C P}^{n}\right) \in 2^{e+2}+\{21,22,23\}$, whereas $\operatorname{Emb}\left(\mathrm{P}^{2 n+1}\right) \leq$ $2^{e+2}+22$ if $e \geq 4$.

(e) For $n=2^{e}+9 \geq 25$, both $\operatorname{Emb}\left(\mathbb{C P}^{n}\right)$ and $\operatorname{Emb}\left(\mathrm{P}^{2 n+1}\right)$ are of the form $2^{e+2}+\{31,32\}$.

Remark 6.4. With the above as indirect evidence, and in order to illustrate its potential usefulness, we observe that an embedding version of the first statement in Fact 6.1 would imply, in view of Theorem 1.1, that the $(4 n-\alpha(n)-1)$-dimensional Euclidean embedding of $\mathbb{C P}^{n}$ (for odd $n>1$ ) in [41] would not only be optimal for $\alpha(n)=2$, but would also produce a corresponding $(4 n-\alpha(n))$-dimensional Euclidean embedding of any lens space $L^{2 n+1}\left(2^{e}\right)$, optimal when $e \geq \alpha(n)=2$, in view of Theorem 1.1.

In the direction of the potential optimality of the embeddings in $[\mathbf{4 1}]$, we observe that the immersion (and consequently embedding) dimension of $\mathbb{C P}^{3}$ is 9 . On the one hand, Remark 6.4 already mentions the embedding $\mathbb{C P}^{3} \subset \mathbb{R}^{9}$. On the other, the normal bundle $\nu$ of a hypothetical codimension- 2 immersion of $\mathbb{C P}^{3}$ would necessarily be the realification of a complex line bundle. Over $\mathbb{C P}^{3}$ this would be of the form $\nu=\left(H^{d}\right)_{\mathbb{R}}$, the realification of the $d$-th power of the Hopf bundle $(d \in \mathbb{Z})$. In particular, the first Pontryagin class of $\nu$ would easily be computed (using for instance [40, Corollary 15.5]) to be $d^{2} a^{2}$, where $a \in H^{2}\left(\mathbb{C P}^{3} ; \mathbb{Z}\right)$ stands for the Euler class of $H$. However this is incompatible with the calculation of the (tangential) Pontryagin class $p_{1}\left(\mathbb{C P}^{3}\right)=4 a^{2}$ in $[\mathbf{4 0}$, page 178$]$ and the fact that $p_{1}\left(\mathbb{C P}^{3}\right)+p_{1}(\nu)=0$ (recall that $\mathbb{C P}^{3}$ is torsion-free).

Remark 6.5. As for the usefulness of a possible embedding analogue of the second statement in Fact 6.1, we remark that this would immediately imply the validity of Theorem 1.2 from the known validity for $e=1$.

Remark 6.6. $\mathrm{P}^{7}$ is an important benchmark giving an exceptional situation both for Fact 6.2 and Theorem 1.1. This manifold is known not to embed in $\mathbb{R}^{8}([\mathbf{2 3}])$, but has best currently known embedding in $\mathbb{R}^{12}([\mathbf{3 4}])$, and even in $\mathbb{R}^{10}$, if the embedding is only required to be piecewise linear $([\mathbf{4 4}])$. It is interesting to observe that a

\footnotetext{
${ }^{6}$ Only projective spaces are considered in this sample; trying to include lens spaces gives an excellent motivation for sharpening the embeddings in Theorem 1.3, and in particular for trying to close up the small gaps indicated in the second paragraph after that theorem.
} 
(topological, at least) embedding

$$
L^{7}\left(2^{e}\right) \subset \mathbb{R}^{10}
$$

could potentially be obtained for any $e$ by quotienting out the action of $\mathbb{Z} / 2^{e}$ both in the fiber and total space of the Hopf fibration $S^{3} \rightarrow S^{7} \rightarrow S^{4}$. Indeed, this produces a fibration $L^{3}\left(2^{e}\right) \rightarrow L^{7}\left(2^{e}\right) \rightarrow S^{4}$ that, when restricted to the hemispheres of $S^{4}$, leads to a splitting of $L^{7}\left(2^{e}\right)$ into two parts, each fiber homeomorphic to $L^{3}\left(2^{e}\right) \times D^{4}$ and, therefore (Remark 2.4), embedding in $\mathbb{R}^{9}$. Following the philosophy in the previous section, if these embeddings were (homotopy) compatible in the common intersection, then there would be a reasonable chance of getting the embedding in (37). Rees' embedding $P^{7} \subset \mathbb{R}^{10}$ would seem to suggest that such compatibility can be attained for $e=1$. But we have not been able to make this idea work for general $e$ (notice that (37) would be fully compatible with the possibilities discussed in Remark 6.4). Unfortunately, the relevance of a possible embedding (37) within the inductive proof of Theorem 1.3 is admittedly limited; the reader will check that the only situation where (37) would produce a better embedding than those described in Theorem 1.3 (besides the case $n=3$ in the second column of Table 1 ) is for improving $(e, n, d)=$ $(\leq 2,7,26)$, the special triples in Theorem 1.3 , to $(e, n, d)=(\leq 2,7,25)$-pretty much as described at the beginning of Remark 4.11.

We have just mentioned the possibility of extending Rees' topological embedding $\mathrm{P}^{7} \subset \mathbb{R}^{10}$ to 7 -dimensional $2^{e}$-torsion lens spaces. In this connection, it is well to keep in mind Sanderson's conjecture in $[\mathbf{4 6}]$ that the smooth embedding dimension of $\mathrm{P}^{7}$ is 11.

Example 6.7. Consider lens spaces $L^{2 n+1}\left(2^{e}\right)$ with $n=2^{t}+1$ for $t \geq 2$ (the case of $t \leq 1$ is described in Remark 2.4). For $e \geq 2$, i.e., high-torsion lens spaces, the 1-dimension gap we leave for $\operatorname{Emb}\left(L^{2 n+1}\left(2^{e}\right)\right)=2^{t+2}+\delta(e)$, with $\delta(e) \in\{2,3\}$, is a shifted version of the known gap for $e=1$ : according to [8] the best current information gives $\operatorname{Emb}\left(\mathrm{P}^{2 n+1}\right)=2^{t+2}+\delta(1)$, with $\delta(1) \in\{1,2\}$.

\section{References}

[1] L. Astey, Geometric dimension of bundles over real projective spaces, Quart. J. Math. Oxford Ser. (2) 31 (1980), no. 122, 139-155.

[2] L. Astey, A cobordism obstruction to embedding manifolds, Illinois J. Math. 31 (1987), no. 2, 344-350.

[3] M.F. Atiyah and F. Hirzebruch, Quelques théorèmes de non-plongement pour les variétés différentiables, Bull. Soc. Math. France 87 (1959), 383-396.

[4] R.R. Bruner, D.M. Davis, and M. Mahowald, Nonimmersions of real projective spaces implied by tmf, in Recent progress in homotopy theory (Baltimore, MD, 2000), Contemp. Math. 293, 45-68, Amer. Math. Soc., Providence, RI (2002).

[5] D.M. Davis, A strong nonimmersion theorem for real projective spaces, Ann. of Math. (2) 120 (1984), no. 3, 517-528.

[6] D.M. Davis, Immersions of projective spaces: a historical survey, in Algebraic topology (Oaxtepec, 1991), Contemp. Math. 146, 31-37, Amer. Math. Soc., Providence, RI (1993). 
[7] D.M. Davis, Embeddings of real projective spaces, Bol. Soc. Mat. Mexicana (3) 4 (1998), no. 1, 115-122.

[8] D.M. Davis, Table of immersions and embeddings of real projective spaces, http://www.lehigh.edu/ dmd1/immtable.

[9] D.M. Davis and M.E. Mahowald, The geometric dimension of some vector bundles over projective spaces, Trans. Amer. Math. Soc. 205 (1975), 295-315.

[10] D.M. Davis and M. Mahowald, Immersions of complex projective spaces and the generalized vector field problem, Proc. London Math. Soc. (3) 35 (1977), no. 2, 333-344.

[11] D.M. Davis and M. Mahowald, Nonimmersions of $R P^{n}$ implied by $t m f$, revisited, Homology, Homotopy Appl. 10 (2008), no. 3, 151-179.

[12] D.M. Davis and V. Zelov, Some new embeddings and nonimmersions of real projective spaces, Proc. Amer. Math. Soc. 128 (2000), no. 12, 3731-3740.

[13] T. Ganea and G. Vranceanu, Topological embeddings of lens spaces, Proc. Cambridge Philos. Soc. 57 (1961), no. 3, 688-690.

[14] S. Gitler and M. Mahowald, The geometric dimension of real stable vector bundles, Bol. Soc. Mat. Mexicana (2) 11 (1966), 85-107.

[15] S. Gitler and M. Mahowald, The immersion of manifolds, Bull. Amer. Math. Soc. 73 (1967), no. 5, 696-700.

[16] S. Gitler and M. Mahowald, Some immmersions of real projective spaces, Bol. Soc. Mat. Mexicana (2) 14 (1969), 9-21.

[17] S. Gitler, M. Mahowald, and R.J. Milgram, Secondary cohomology operations and complex vector bundles, Proc. Amer. Math. Soc. 22 (1969), 223-229.

[18] J. González, Connective $K$-theoretic Euler classes and non-immersions of $2^{k}$-lens spaces, J. London Math. Soc. (2) 63 (2001), no. 1, 247-256.

[19] J. González, A generalized Conner-Floyd conjecture and the immersion problem for low 2-torsion lens spaces, Topology 42 (2003), no. 4, 907-927.

[20] J. González and P. Landweber, Symmetric topological complexity of projective and lens spaces, Algebr. Geom. Topol. 9 (2009), no. 1, 473-494.

[21] J. González and T.A. Shimkus, On the immersion problem for $2^{r}$-torsion lens spaces, Topology Appl. 145 (2004), no. 1-3, 261-275.

[22] A. Haefliger, Plongements différentiables dans le domaine stable, Comment. Math. Helv. 37 (1962/1963), 155-176.

[23] W. Hantzsche, Einlagerung von Mannigfaltigkeiten in euklidische Räume, Math. Z. 43 (1938), no. 1, 38-58.

[24] A. Hatcher, Algebraic Topology, Cambridge University Press, Cambridge, 2002.

[25] M.W. Hirsch, The imbedding of bounding manifolds in Euclidean space, Ann. of Math. (2) 74 (1961), 494-497.

[26] W.C. Hsiang and R.H. Szczarba, On the embeddability and nonembeddability of certain parallelizable manifolds, Bull. Amer. Math. Soc. 69 (1963), no. 4, $534-536$. 
[27] I.M. James, On the immersion problem for real projective spaces, Bull. Amer. Math. Soc. 69 (1963), no. 2, 231-238.

[28] N. Kitchloo and W.S. Wilson, The second real Johnson-Wilson theory and nonimmersions of $R P^{n}$, Homology, Homotopy Appl. 10 (2008), no. 3, 223-268.

[29] N. Kitchloo and W.S. Wilson, The second real Johnson-Wilson theory and nonimmersions of $R P^{n}$, Part II, Homology, Homotopy Appl. 10 (2008), no. 3, 269-290.

[30] T. Kobayashi and M. Sugawara, Note on KO-rings of lens spaces mod $2^{r}$, Hiroshima Math. J. 8 (1978), no. 1, 85-90.

[31] J. Levine, Imbedding and immersion of real projective spaces, Proc. Amer. Math. Soc. 14 (1963), no. 5, 801-803.

[32] B.H. Li and Z.Z. Tang, Codimension 1 and 2 immersions of lens spaces, in Differential geometry and topology (Tianjin, 1986-1987), Lecture Notes in Math. 1369, 152-163, Springer-Verlag, New York, 1989.

[33] M. Mahowald, On the embeddability of the real projective spaces, Proc. Amer. Math. Soc. 13 (1962), 763-764.

[34] M. Mahowald, On obstruction theory in orientable fiber bundles, Trans. Amer. Math. Soc. 110 (1964), 315-349.

[35] M. Mahowald, The Metastable Homotopy of $S^{n}$, Mem. Amer. Math. Soc. 72, Amer. Math. Soc., Providence, RI, 1967.

[36] M. Mahowald and R.J. Milgram, Embedding real projective spaces, Ann. of Math. (2) 87 (1968), no. 3, 411-422.

[37] W.S. Massey, On the imbeddability of the real projective spaces in Euclidean space, Pacific J. Math. 9 (1959), no. 3, 783-789.

[38] W.S. Massey and F.P. Peterson, On the dual Stiefel-Whitney classes of a manifold, Bol. Soc. Mat. Mexicana (2) 8 (1963), 1-13.

[39] R.J. Milgram, Immersing projective spaces, Ann. of Math. (2) 85 (1967), no. 3, 473-482.

[40] J.W. Milnor and J.D. Stasheff, Characteristic Classes, Annals of Math. Studies, 76, Princeton University Press, Princeton, NJ, 1974.

[41] A. Mukherjee, Embedding complex projective spaces in Euclidean space, Bull. London Math. Soc. 13 (1981), no. 4, 323-324.

[42] R. Nakagawa, Embeddings of projective spaces and lens spaces, Sci. Rep. Tokyo Kyoiku Daigaku Sect. A 9 (1967), 170-175.

[43] R. Nakagawa, Note on embeddings of lens spaces, Proc. Japan Acad. 45 (1969), 107-108.

[44] E. Rees, Embeddings of real projective spaces, Topology 10 (1971), 309-312.

[45] R. Rigdon, $p$-equivalences and embeddings of manifolds, J. London Math. Soc. (2) 2 (1975), no. 2, 233-244.

[46] B.J. Sanderson, Immersions and embeddings of projective spaces, Proc. London Math. Soc. (3) 14 (1964), 137-153. 
[47] T.A. Shimkus, Some new immersions and nonimmersions of $2^{r}$-torsion lens spaces, Bol. Soc. Mat. Mexicana (3) 9 (2003), no. 2, 339-357. Erratum published in Bol. Soc. Mat. Mexicana (3) 10 (2004), no. 2, 245-247.

[48] D. Sjerve, Vector bundles over orbit manifolds, Trans. Amer. Math. Soc. 138 (1969), 97-106.

[49] B. Steer, On the embedding of projective spaces in Euclidean spaces, Proc. London Math. Soc. (3) 21 (1970), 489-501.

[50] E. Thomas, Embedding manifolds in Euclidean space, Osaka J. Math. 13 (1976), no. $1,163-186$.

Jesús González jesus@math.cinvestav .mx

Mathematics Department, CINVESTAV-IPN, Apartado Postal 14-740, Mexico City, 07000, Mexico (on sabbatical leave visiting CIMAT, Guanajuato, Mexico)

Peter Landweber landwebe@math.rutgers.edu

Department of Mathematics, Rutgers University, 110 Frelinghuysen Rd, Piscataway, NJ 08854-8019, USA

Thomas Shimkus shimkust2@scranton.edu

Department of Mathematics, University of Scranton, Scranton, PA 18510-4666, USA 